Sección Básica / Basic

Artículo de investigación / Research paper

\title{
Efecto de la temperatura en la expresión fenotípica de caracteres de las manchas costales de Nyssorhynchus triannulatus (Diptera: Culicidae: Anophelinae)
}

\author{
Temperature effect on the phenotypic expression of characters of costal spots \\ Nyssorhynchus triannulatus (Diptera: Culicidae: Anophelinae) \\ RANULFO GONZÁLEZ OBANDO'; HEIBER CÁRDENAS HENAO²; \\ OMAR ALEJANDRO MARÍN-LONDOÑO ${ }^{3}$
}

\begin{abstract}
${ }^{1}$ Ph. D. Departamento de Biología, Facultad de Ciencias Naturales y Exactas, Universidad del Valle, Santiago de Cali, Colombia, ranulfo.gonzalez@ correounivalle.edu.co, https://orcid.org/0000-0003-4047-8677. ${ }^{2} \mathrm{Ph}$. D. Departamento de Biología, Facultad de Ciencias Naturales y Exactas, Universidad del Valle, Santiago de Cali, Colombia, heiber.cardenas@correounivalle.edu.co, https://orcid.org/0000-0003-2823-8443. ${ }^{3}$ B. Sc. Programa de Postgrado en Biología, Facultad de Ciencias Naturales y Exactas, Universidad del Valle, Santiago de Cali, Colombia, omarmarin@correounivalle.edu.co, https://orcid. org/0000-0002-5172-4960.
\end{abstract}

\begin{abstract}
Autor para correspondencia
Ranulfo González Obando. Ph. D. Departamento de Biología, Facultad de Ciencias Naturales y Exactas, Universidad del Valle, Santiago de Cali, Colombia, ranulfo.gonzalez@correounivalle.edu.co, https://orcid.org/0000-0003-40478677.
\end{abstract}

\section{Citación sugerida}

GONZÁLEZ OBANDO, R.; CÁRDENAS HENAO, H.; MARÍN-LONDOÑO, O. A 2021. Efecto de la temperatura en la expresión fenotípica de caracteres de las manchas costales de Nyssorhynchus triannulatus (Diptera: Culicidae: Anophelinae). Revista Colombiana de Entomología 47 (1): e8456. https://doi. org/10.25100/socolen.v47i1.8456

Recibido: 28-ago-2019

Aceptado: 09-sep-2020

Publicado: 13-abr-2021

Revista Colombiana de Entomología ISSN (Print): 0120-0488

ISSN (On Line): 2665-4385

https://revistacolombianaentomologia.univalle.edu.co

Open access

(c) (i) (5) (2) BY-NC-SA 4.0

Publishers: Sociedad Colombiana de Entomología SOCOLEN (Bogotá, D. C., Colombia) https://www.socolen.org.co

Universidad del Valle (Cali, Colombia)

https://www.univalle.edu.co

(C) 2021 Sociedad Colombiana de Entomología SOCOLEN y Universidad del Valle - Univalle
Resumen: En el presente trabajo, mediante morfometría tradicional, se evaluó el efecto de la temperatura sobre la expresión fenotípica del tamaño, el alargamiento (largo/ancho) y las manchas costales de las alas entre familias, progenies y sexos, en Nyssorhynchus triannulatus del sur de Colombia. En las progenies de 11 familias, criadas a 20,24 y $28{ }^{\circ} \mathrm{C}$, las alas presentaron cuatro patrones de manchas costales. El patrón I estuvo presente en las tres temperaturas como el más frecuente. Dentro de familias la tasa promedio largo/ancho de las alas fue significativamente mayor en machos respecto a hembras, mientras que las variaciones por sexo de esta característica fueron similares. Entre las familias, la temperatura influyó de manera variable sobre los tamaños de las alas y las manchas costales. En los componentes de la variación fenotípica, no se detectó efecto significativo de la temperatura de crianza sobre la longitud del ala y varios de los caracteres analizados, pero la tasa longitud de la mancha subcostal clara sobre la longitud de la mancha sectorial oscura distal (SCP/DSD) fue la más sensible a los cambios de temperatura. El aporte genético del tamaño del ala y la longitud de los caracteres medidos, así como el efecto de las familias (origen) en la variación fenotípica, fue significativo en casi todos los caracteres analizados. El efecto de los sexos sobre la variación fenotípica de los caracteres estudiados solo fue significativo para algunos de ellos y se observaron pocas interacciones significativas entre los tres factores analizados (temperatura, familia y sexo) con efecto bajo sobre la variación fenotípica.

Palabras clave: Genética, morfología, mosquitos, morfometría tradicional, Nyssorhynchus triannulatus, Anophelinae, Colombia.

Abstract: In this study, was evaluated through traditional morphometry, the effect of temperature on the phenotypic expression of size, elongation (length/width) and costal wing spots among families, progenies and sexes in populations of Nyssorhynchus triannulatus from southern Colombia. In the progenies of 11 families, obtained at 20,24 and $28{ }^{\circ} \mathrm{C}$ the wings showed four costal spots patterns. Pattern I was present in all three breeding temperatures with the highest frequency. Within families, the average length/width of wing ratio was significantly higher in males than females, while the variation by sex of this character was similar. Among the families, the temperature had variable influence on the sizes of wings and costal spots. Within the components of phenotypic variation, the breeding temperature had no significant detectable effect on the length of the wing and several of the analyzed characters; however, the proportion of the subcostal pale spot length over the length of the distal sector dark spot (SCP/DSD) was the most sensitive to temperature changes. The genetic contribution of the wing size and the measured characters length, as well as the effect of the families (origin) on the phenotypic variation, was significant for almost all the characters analyzed. The effect of the sexes on the phenotypic variation of the studied characters was significant only for some characters, while there were few significant interactions between the three factors analyzed (temperature, family and sex) with a low effect on the phenotypic variation.

Keywords: Genetics, morphology, mosquitoes, traditional morphometry, Nyssorhynchus triannulatus, Anophelinae, Colombia. 


\section{Introducción}

En taxonomía, es indudable que los caracteres discretos facilitan el proceso de las diagnosis específicas de los organismos biológicos, sin embargo, frecuentemente es necesario hacer uso de caracteres continuos, especialmente cuando se trata de especies estrechamente relacionadas. Esto último, es el caso de especies de mosquitos Nyssorhynchus, considerados aquí según la propuesta de Foster et al. (2017), quienes elevaron a género a este y otros subgéneros de Anopheles. En estos, cuando se trata de situaciones taxonómicas confusas, se emplean marcadores moleculares, de los cuales el código de barras de $\mathrm{ADN}$ (COI) es uno de los más usados debido a la disponibilidad de cebadores universales que amplifican a través de un amplio rango de especies, su baja divergencia intraespecífica pero alta divergencia interespecífica (Bourke et al. 2018) (para Anophelinae, ver González et al. 2010; Gómez et al. 2015; Scarpassa et al. 2016; Saraiva et al. 2018). Sin embargo, no siempre se dispone de la facilidad logística de laboratorios para realizar estas pruebas moleculares y, por lo tanto, la información morfológica sigue teniendo un gran peso en la diferenciación de las especies de Anophelinae. En las caracterizaciones morfológicas, es común el uso de tasas que relacionan caracteres próximos, especialmente de las alas y patas posteriores (Faran 1980; Faran y Linthicum 1981; Linthicum 1988; González Obando y Carrejo Gironza 2009). Sin embargo, por la naturaleza de los datos discontinuos, especialmente para especies de un mismo género, subgénero, grupos o complejo de especies próximas, no se presenta una discontinuidad clara entre las tasas empleadas, dado que estas son variables entre los individuos de una misma población e incluso entre las progenies de una misma familia.

Debido a lo anterior, para la determinación específica del género Nyssorhynchus, generalmente se hace uso de información morfológica combinada y asociada de larvas, hembras y genitalia de machos, obtenidos a partir de la producción de isofamilias. Pero, no siempre se puede tener disponibilidad de estos estados y lo más práctico es la obtención de hembras. En estas, la mayor parte de la información diagnóstica de especies se logra principalmente a partir, de las manchas claras y oscuras del área costal de las alas. En el proceso de búsqueda de métodos apropiados para la diferenciación de especies a partir de la información de las alas de las hembras, el análisis de la forma de estas ha sido una opción. El empleo de morfometría geométrica ha tenido relativa utilidad en la diferenciación de especies crípticas de Anopheles (Petrarca et al. 1998; Calle et al. 2002; Gómez et al. 2013; Pacheco et al. 2017; Pacheco-Gómez et al. 2018). Sin embargo, la morfometría tradicional parece ser una herramienta de fácil aplicación por personal entrenado, bajo costo y gran potencial para la exploración de la diversidad de especies (Cazorla 2009). Además, ha sido de gran utilidad para esclarecer problemas taxonómicos en especies de difícil diagnóstico, describir variaciones morfológicas en poblaciones a diferentes altitudes y diferenciar poblaciones provenientes de diferentes ecorregiones (Belen et al. 2004; Bustamante et al. 2004; Dantur Juri et al. 2011; Dos Santos Silva et al. 2012; Rodríguez-Zabala et al. 2016). Por otro lado, para entender cómo se regula el desarrollo de los caracteres de un organismo hasta alcanzar un fenotipo ideal, la morfometría tradicional es una de las herramientas de estudio, que utiliza métodos estadísticos multivariados a colecciones de varia- bles de tamaño o conformación, en la que se definen mediciones con el fin de registrar aspectos biológicos significativos del organismo.

En el estudio de la variación morfológica, los cambios fenotípicos dependen de factores como $\mathrm{pH}$, humedad, temperatura, fotoperiodo, estacionalidad, entre otros (Müller 2007), lo cual para especies de Culicidae se traduce en plasticidad fenotípica. De acuerdo con Motoki et al. (2012) las diferencias ambientales entre ecorregiones podrían conducir a diferenciación fenotípica de poblaciones de Anophelinae, expresada en cambios químicos, fisiológicos, del desarro1lo, morfológicos, de conducta, entre otros (Fusco y Minelli 2010).

En especies de Anophelinae, las alas presentan una serie de caracteres fenotípicos variables analizados por morfometría tradicional, ya sea dentro y entre poblaciones y progenies de isofamilias (Hribar 1995; Fajardo Ramos et al. 2008; Pacheco et al. 2017; Pacheco-Gómez et al. 2018) y entre especies (Rosa-Freitas et al. 1990; Rubio-Palis 2000). Mediante este método, la información de las alas puede ser usada para estudiar el aporte del componente genético a la variación fenotípica. La variabilidad en el tamaño del ala y en la longitud de las manchas costales, puede ser utilizada para analizar el aporte del componente genético a la variación fenotípica, el grado de canalización, de integración morfológica y de asimetría fluctuante presentes en respuesta a cambios ambientales como la temperatura. Para obtener información de estos caracteres, es fundamental la realización de experimentos de ecofisiología, donde se pueda establecer control de las variables a evaluar (por ejemplo, temperatura), manteniendo constantes el resto de las variables (humedad relativa, fotoperiodo, alimentación, densidad larval, entre otras).

Considerando lo anterior, más allá de lo meramente taxonómico, el uso de métodos morfométricos puede ser también aprovechado para medir el efecto ambiental sobre la expresión fenotípica de caracteres morfológicos, pero hasta el momento, no se conocen trabajos publicados con los mosquitos como modelos biológicos para estudiar cómo se descompone la variación fenotípica de la forma, el tamaño y los patrones de manchas por efecto de cambios ambientales como la temperatura. En el presente trabajo, mediante morfometría tradicional, se evaluó el efecto de la temperatura en la expresión fenotípica del tamaño, alargamiento de las alas y longitud de las manchas costales entre familias, progenies y sexos, utilizando como organismo modelo a Nyssorhynchus triannulatus (Neiva y Pinto, 1922). Mediante este método, en esta especie se estimó los aportes de la variación ambiental (efecto de la temperatura), del componente genético (variación entre familias) y la interacción genotipo-ambiente sobre la variabilidad fenotípica de los caracteres descritos arriba.

\section{Materiales y métodos}

Las hembras de anofelinos fueron recolectadas en el municipio de Puerto Asís, Putumayo, Colombia (0³0'02"N 76²9'56"O) sobre cebo humano protegido y con búsqueda activa del mosquito en reposo en el intradomicilio y vegetación del peridomicilio. Los mosquitos se recolectaron entre las 18:00 y 24:00 horas, de tres a cinco días consecutivos en dos salidas (en noviembre 2018 y abril 2019) con un aspirador bucal y se depositaron en recipientes plásticos de 300 $\mathrm{ml}$, hasta un máximo de 25 a 30 individuos/recipiente. Se les 
transportó en cajas térmicas al Laboratorio de Entomología de la Universidad del Valle, en Santiago de Cali, con suministro de azúcar diluida en agua en un algodón, para obtener isofamilias y realizar la identificación respectiva.

Para la obtención de las progenies, cada una de las hembras recolectadas se colocó en una cámara de ovipostura. Cada cámara estuvo compuesta de un vaso plástico desechable de $200 \mathrm{~cm}^{3}$ de capacidad, con una capa de algodón húmedo en el fondo y sobre éste, papel filtro blanco acondicionado para la oviposición. Cada hembra se alimentó con sangre de cobayo y agua azucarada. Las cámaras se almacenaron en cajas térmicas acondicionadas para el mantenimiento de alta humedad relativa y una temperatura promedio de $24{ }^{\circ} \mathrm{C}$. A partir del segundo día se realizaron observaciones diarias para identificar posturas de huevos. Cada postura/hembra se incubó separadamente en bandejas plásticas de $25 \times 30 \times 5$ $\mathrm{cm}^{3}$, mantenidas en una cámara ambientada SANYO, modelo MLR $351 \mathrm{H}$, regulada en el tiempo a 20, 24 y $28^{\circ} \mathrm{C}$. En cada temperatura, las larvas se criaron en agua reposada hasta alcanzar el estado adulto, manteniendo constantes la humedad relativa, la alimentación y un fotoperiodo de 12 horas luz/ noche. Las larvas de los estadios I y II se alimentaron con levadura de cerveza y las de estadios III y IV con una combinación de polvo de alimento para conejos y levadura de cerveza. Las pupas se individualizaron en vasos plásticos de $100 \mathrm{~cm}^{3}$ cubiertos con tul hasta obtener los respectivos adultos. Los adultos se alimentaron con solución azucarada por dos días $\mathrm{y}$ posteriormente se sacrificaron en frio y se preservaron en alfileres entomológicos. Cada ejemplar se rotuló con el código de su respectiva postura. Nyssorhynchus triannulatus s.l. se identificó por los caracteres diagnósticos de las hembras y posteriormente se verificó a partir de la genitalias de los machos obtenidos de cada isofamilia. Las genitalias de machos se procesaron y preservaron en placas, siguiendo el método de González Obando y Carrejo Gironza (2009). Esta especie podría corresponder al linaje nombrado por Moreno et al. (2013) como Nys. triannulatus E, dado que el sitio de muestreo se ubicó próximo a poblaciones amazónicas de Ecuador y del departamento colombiano de Amazonas. Sin embargo, es importante tener presente que Nyssorhynchus triannulatus es considerado un complejo en el cual se reconocen almenos tres especies hermanas, Nys. triannulatus s.s. (Neiva y Pinto), Nys. halophylus (Silva do Nascimento y Lourenço-de-Oliveira 2002) y Nys. triannulatus C (Silva-do-Nascimento et al. 2006; Moreno et al. 2013). Este último no ha sido caracterizado morfológicamente, pero en lo que respecta a las dos primeras, se pueden diferenciar por caracteres morfológicos de los genitales de machos, formas setales y aparato espiracular de larvas de cuarto estadio y aspecto general del huevo (Silva do Nascimento y Lourenço-de-Oliveira 2002).

Las alas derechas e izquierda y una de las patas posteriores de cada ejemplar se preservaron en una placa portaobjeto utilizando un cubreobjeto de $22 \mathrm{~mm}$, fijado con bálsamo de Canadá. La asignación y medición de las diferentes manchas costales se realizó siguiendo la nomenclatura propuesta por Wilkerson y Peyton (1990). Los análisis por morfometría tradicional se basaron en un registro fotográfico de alas (aproximadamente 20 fotografías/ala) empleando una cámara fotográfica digital Canon Eos Rebel T5i adaptada a un microscopio Nikon modelo E-Ci, y el software Helicon Focus 5.3. (Helicon Soft Ltd.). Después, se procedió a la toma de medidas con ayuda del programa CorelDRAW Graphics Suite X7, midiendo la longitud, el ancho y los tamaños de las manchas costales del ala. Se usó la siguiente nomenclatura durante la toma de medidas las alas (Fig. 1): Prehumeral oscura (PHD), Humeral clara (HP), Humeral oscura (HD), Presector claro (PSP), Presector oscuro (PSD), Sector claro (SP), Sector oscuro proximal (PRSD), Sector claro accesorio (ASP), Sector oscuro distal (DSD), Subcostal clara (SCP), Preapical oscura (PD), Preapical clara (PP), Apical oscura (AD), Apical clara (AP).

En las patas posteriores, se midió la longitud del tarsómero dos (ta- $\left.\mathrm{III}_{2}\right)$ y área oscura basal del mismo. También, se calcularon las tasas alargamiento (longitud/ancho), SCP/DSD (longitud de la mancha subcostal clara)/(longitud de la mancha sectorial oscura distal) y HP/PHD (longitud de la mancha clara humeral)/(longitud de la mancha oscura prehumeral). Además, se consideró la suma de las longitudes de las manchas del sector claro (SP), sector oscuro proximal (PRSD) y sector claro accesorio (ASP), SP + PRSD + ASP. Para las patas posteriores se usó la tasa de la longitud de la parte oscura basal del tarsómero dos ( ta-III $_{2}$ ) sobre la longitud del tarsómero. Los datos se tabularon en una hoja de cálculo del programa Microsoft Excel para Office 365. Con el fin de aproximar la distribución de los datos a una distribución normal, previo a los análisis estadísticos, se procedió a su transformación usando la fórmula de Freeman y Tukey (1950). Para tasas se utilizó la ecuación de Kihlberg et al. (1972). Los análisis descriptivos presentan el promedio, la desviación estándar y valores mínimos y máximos.

Para probar si la temperatura influyó en la variación de los rasgos estudiados se escogieron tres niveles del factor temperatura $\left(20,24\right.$ y $\left.28^{\circ} \mathrm{C}\right)$ y se sometieron las progenies de cuatro isofamilias a $20^{\circ} \mathrm{C}$ y $24{ }^{\circ} \mathrm{C}$ y tres a $28{ }^{\circ} \mathrm{C}$. Para estimar el efecto del componente genético sobre la variación fenotípica de los rasgos estudiados se asumió que las hembras grávidas recolectadas fueron monoandricas y que el parentesco entre la descendencia de una sola hembra es de 0,5 (Tripet et al. 2003; Shutt et al. 2010; Richardson et al. 2015). Es decir, las progenies derivadas de cada hembra grávida fueron mayoritariamente hermanos completos. En consecuencia, se estimó la heredabilidad en sentido amplio $\left(\mathrm{H}^{2}\right)$ utilizando un modelo de hermanos completos, como lo describen Falconer y Mackay (1996) con algunas modificaciones. Por lo tanto, se consideró a las isofamilias como un segundo factor (genético) que afecta la variación fenotípica de los caracteres estudiados.

Por último, se incluyó como un tercer factor, el sexo, dado que dentro de las progenies por familia se obtienen hembras y machos desarrollados bajo las mismas condiciones dentro de la isofamilia. Con este factor se evaluó si las progenies presentaban dimorfismo sexual para los caracteres estudiados, usando análisis de variación (ANDEVA). Se probó la hipótesis en la que se consideró que los promedios para la longitud, la tasa largo/ancho y las longitudes de las manchas costales del ala derecha y la tasa $\mathrm{PB} /$ ta-III ${ }_{2}$, dentro de isofamilias son iguales entre progenies hembras y machos, asumiendo que las variaciones de las progenies hembras y machos son desconocidas pero similares. Para el contraste de medias se usó la prueba t-Student; posteriormente se realizaron comparaciones múltiples con corrección de Bonferroni para probar cada hipótesis individual bilateral $\left(H_{0}=\mu_{1}-\mu_{2}=0\right)$ en un nivel significativo de $\alpha / \mathrm{m}$, donde $\alpha$ es el nivel de significancia deseado $(0,05)$ y $\mathrm{m}$ es el número de hipótesis (Bland y Altman 1995).

Dado que, a partir del modelo general ninguno de los análisis de variación mostró efectos de interacción significativamente diferentes de cero, el modelo se ajustó a uno de tres factores sin interacción: $y_{i j k}=\mu+\alpha_{i}+\beta_{j}+\gamma_{k}+\varepsilon_{i j k}$. Para probar 
este modelo, se usaron análisis lineales generalizados mixtos, considerando como variable dependiente las medidas morfométricas y como fuentes de variación la temperatura, la procedencia de las progenies (familias) y el sexo. También se usó la prueba de Levene (1960) W, para evaluar la igualdad de las variaciones para cada una de las variables analizadas, calculada para dos o más grupos (3 temperaturas, 11 familias).

\section{Resultados}

En las 11 familias de Nys. triannulatus, criadas en las tres temperaturas, se registraron 148 ejemplares ( 98 hembras y 50 machos) (Tabla 1). Solo cuatro patrones fueron observados, dos a $20^{\circ} \mathrm{C}$, tres a $24^{\circ} \mathrm{C}$ y cuatro a $28^{\circ} \mathrm{C}$. El patrón I correspondió a la presencia de las manchas costales nombradas en la nomenclatura propuesta por Wilkerson y Peyton (1990) (Fig. 1A). La no observación de la accesoria clara (ASP) definió los patrones II y III. El patrón II correspondió a la extensión del sector claro (SP) con desaparición del sector oscuro proximal (PRSD) y al menos parte de la ASP, es decir que ésta comprende un área clara grande, la fusión de las manchas SP + PRSD + ASP (Fig. 1B). El patrón III (Fig. 1C) se identificó por la ausencia de la mancha ASP la cual fue ocupada por extensiones del sector oscuro (SD); en este caso, SP se observó más pequeña que en el patrón II y algo similar a la del patrón I. El patrón IV ocurrió cuando el sector claro (SP) fue ocupado por extensiones del presector oscuro (PSD) y el sector oscuro proximal (PRSD) (Fig. 1D), es decir que la ASP sigue siendo observable, pero el sector claro no. Tanto en hembras como en machos, el patrón costal I estuvo presente en las tres temperaturas evaluadas y fue el más frecuente (Fig. 2), sobre todo a $24{ }^{\circ} \mathrm{C}$, donde alrededor del $95 \%$ de las progenies presentaron este tipo de alas, o sea que pocas veces se observó fusión de algunas de las manchas costales, o por lo menos ausencia de la accesoria clara (ASP). El tipo II también estuvo presente en las tres temperaturas, aunque no en los machos de especímenes criados a $28^{\circ} \mathrm{C}$. El patrón III solo se observó en ejemplares obtenidos a 24 y $28^{\circ} \mathrm{C}$, mientras que el patrón IV solo se observó a $28^{\circ} \mathrm{C}$.

El porcentaje esperado de patrones II + III + IV fue 14,9\% en cualquiera de las tres temperaturas. En ambos sexos, el porcentaje observado a $20{ }^{\circ} \mathrm{C}$ fue similar al esperado (hembras $=17,9 \%$; machos $=13,3 \%$ ); a $24^{\circ} \mathrm{C}$, fue menor que el esperado (hembras $=5,1 \%$; machos $=4,3 \%$ ) mientras que a $28{ }^{\circ} \mathrm{C}$ se incrementó significativamente en hembras y fue similar en machos (hembras $=32,3 \%$; machos $=16,7 \%$ ). La Figura 2 muestra claramente esta tendencia.

La longitud de las alas derechas de progenies hembras y machos, mostró tamaños muy variables entre familias de individuos criados a la misma temperatura (Tabla 2). En hembras, las variaciones a $20{ }^{\circ} \mathrm{C}$, varió entre 2,13 y $3,22 \mathrm{~mm}$ $\left(\bar{X}_{H}=2,772 \pm 0,389 \mathrm{~mm}\right)$, a $24{ }^{\circ} \mathrm{C}$ entre 2,30 y $3,20 \mathrm{~mm}$ $\left(\bar{X}_{H}=2,737 \pm 0,258 \mathrm{~mm}\right)$ y a $28{ }^{\circ} \mathrm{C}$ entre 2,22 y $3,17 \mathrm{~mm}$ $\left(\bar{X}_{H}=2,644 \pm 0,274 \mathrm{~mm}\right)$. En las progenies machos, a $20^{\circ} \mathrm{C}$ varió entre 2,24 y $3,45 \mathrm{~mm}\left(\bar{X}_{M}=2,711 \pm 0,418 \mathrm{~mm}\right)$, a $24{ }^{\circ} \mathrm{C}$ entre 2,40 y $3,02 \mathrm{~mm}\left(\bar{X}_{M}=2,670 \pm 0,204 \mathrm{~mm}\right)$ y a $28^{\circ} \mathrm{C}$ entre 2,21 y $2,97 \mathrm{~mm}\left(\bar{X}_{M}=2,560 \pm 0,229 \mathrm{~mm}\right)$.

La comparación de la longitud promedio de las alas derechas de las progenies hembras $(\mathrm{H})$ y machos $(\mathrm{M})$ con respecto a la temperatura, no mostró diferencias significativas en ninguna de las tres comparaciones. En hembras, los valores de t-student para las parejas $20-24^{\circ} \mathrm{C}, 20-28{ }^{\circ} \mathrm{C}$ y $24-28{ }^{\circ} \mathrm{C}$, fueron respectivamente: $0,44(\mathrm{p}=0,6619), 1,43(\mathrm{p}=0,1589)$ y $1,42(p=0,1615)$ mientras que en machos fueron 0,39 $(\mathrm{p}=0,6992), 0,81(\mathrm{p}=0,4264)$ y $1,42(\mathrm{p}=0,1653)$.

La longitud promedio de las alas en las progenies hembras fue significativamente mayor que la de machos en las familias $1-4$ criadas a $20{ }^{\circ} \mathrm{C}$, en la familia 5 criada a $24{ }^{\circ} \mathrm{C}$ y en la familia 11 criada a $28^{\circ} \mathrm{C}(6 / 11=54,5 \%)$ (Tabla 3$)$. Lo contrario se observó para las familias 6 y 7 criadas a $24^{\circ} \mathrm{C}$ $(2 / 11=18,2 \%)$.

No hubo diferencias significativas en la longitud promedio de progenies hembras y machos en la familia 8 criada a 24 ${ }^{\circ} \mathrm{C}$ y en las familias 9 y 10 criadas a $28^{\circ} \mathrm{C}(3 / 11=27,3 \%)$.

Tabla 1. Patrones de manchas costales y frecuencias observadas en progenies de Nyssorhynchus triannulatus, criados en cámaras ambientales a tres temperaturas diferentes.

\begin{tabular}{cccccccc}
\hline \multirow{2}{*}{$\begin{array}{c}\text { Temperatura } \\
\left({ }^{\circ} \mathbf{C}\right)\end{array}$} & Patron & \multicolumn{2}{c}{ Hembras } & \multicolumn{2}{c}{ Machos } & \multicolumn{2}{c}{ Total } \\
\cline { 2 - 8 } & Costal & N & \% & N & \% & N & \% \\
\hline \multirow{2}{*}{20} & I & 23 & 82,1 & 13 & 86,7 & 36 & 83,7 \\
& II & 5 & 17,9 & 2 & 13,3 & 7 & 16,3 \\
& Subtotal & 28 & 100 & 15 & 100 & 43 & 100 \\
\hline \multirow{2}{*}{24} & I & 37 & 94,8 & 22 & 95,7 & 59 & 95,2 \\
& II & 1 & 2,6 & 1 & 4,3 & 2 & 3,2 \\
& III & 1 & 2,6 & 0 & 0,0 & 1 & 1,6 \\
& Subtotal & 39 & 100 & 23 & 100 & 62 & 100 \\
\hline \multirow{2}{*}{28} & I & 21 & 67,7 & 10 & 83,3 & 31 & 72,1 \\
& II & 2 & 6,5 & 0 & 0,0 & 2 & 4,6 \\
& III & 4 & 12,9 & 1 & 8,3 & 5 & 11,6 \\
& IV & 4 & 12,9 & 1 & 8,3 & 5 & 11,6 \\
& Subtotal & 31 & 100 & 12 & 100 & 43 & 100 \\
\hline
\end{tabular}



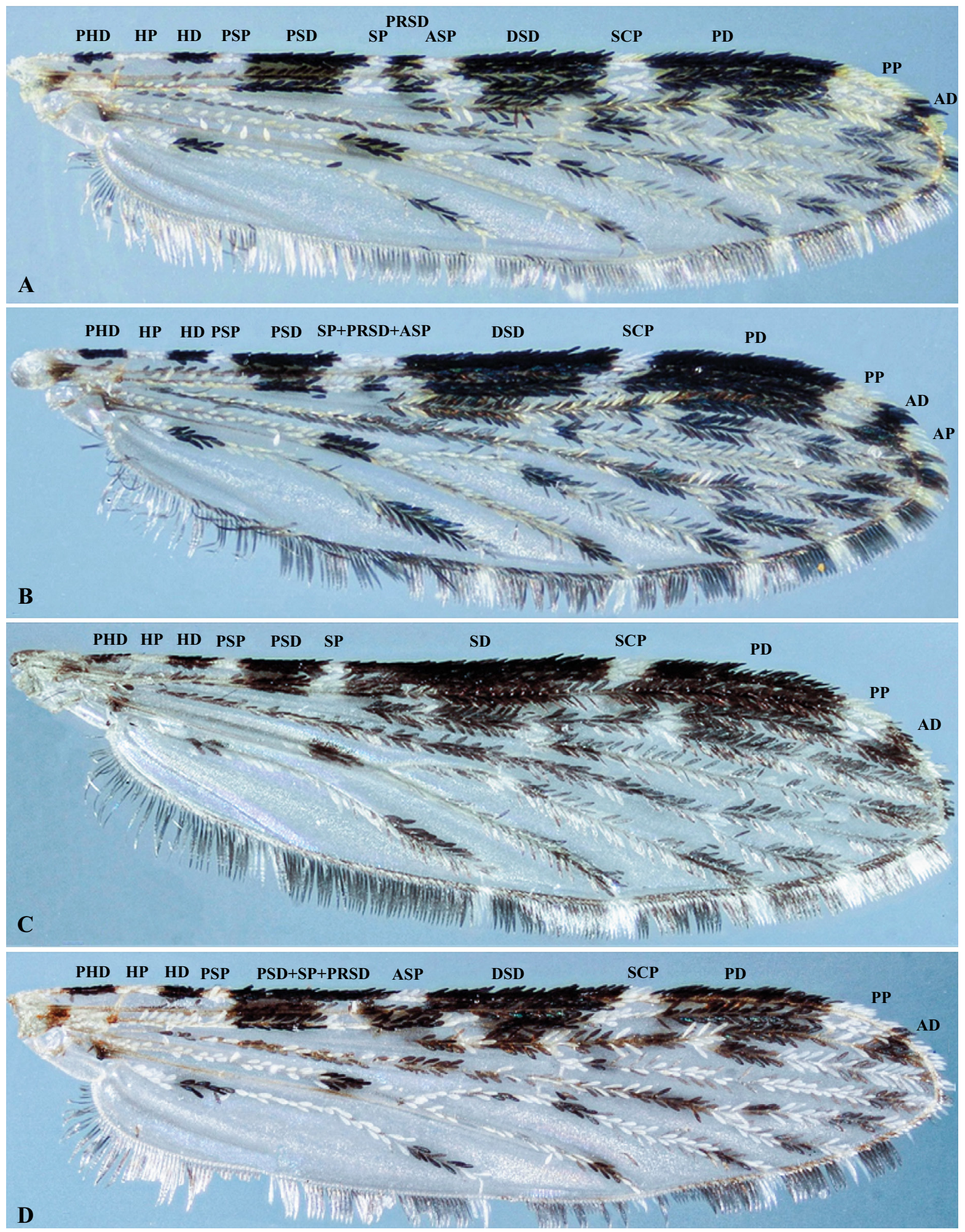

Figura 1. Patrones costales observados en progenies de Nyssorhynchus triannulatus criados en cámaras ambientales a tres temperaturas diferentes. A. Tipo I (Sin fusión de manchas costales). B. Tipo II (SP + PRSD + ASP). C. Tipo III (ausencia de ASP). D. Tipo IV (PSD + SP + PRSD). Nomenclatura: Prehumeral oscura (PHD), Humeral clara (HP), Humeral oscura (HD), Presector claro (PSP), Presector oscuro (PSD), Sector claro (SP), Sector oscuro proximal (PRSD), Sector claro accesorio (ASP), Sector oscuro distal (DSD), Subcostal clara (SCP), Preapical oscura (PD), Preapical clara (PP), Apical oscura (AD), Apical clara (AP). 


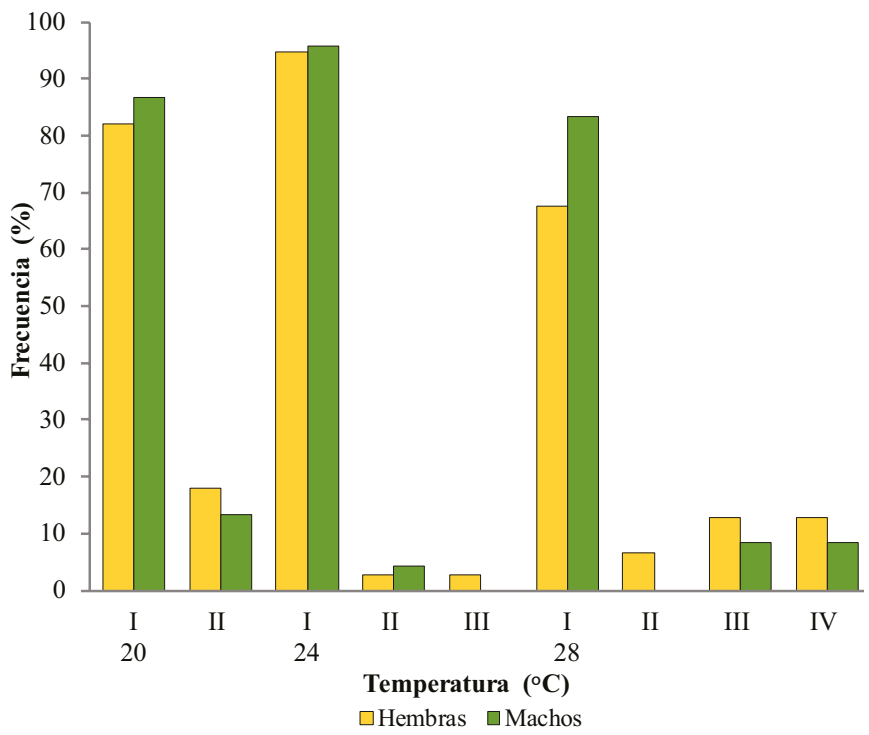

Figura 2. Frecuencias de los patrones de manchas costales observados en hembras $(\mathrm{N}=98)$ y machos $(\mathrm{N}=50)$ de progenies de Nyssorhynchus triannulatus, criados en cámaras ambientales a tres temperaturas.

Tampoco se presentaron diferencias significativas en la longitud promedio de progenies hembras y machos en la familia 8 criada a $24^{\circ} \mathrm{C}$ y en las familias 9 y 10 criadas a $28^{\circ} \mathrm{C}$ $(3 / 11=27,3 \%)$.

La prueba de Levene (1960) W, utilizada para evaluar la igualdad de las variaciones en la longitud del ala, mostró que las progenies hembras y machos criadas a $20^{\circ} \mathrm{C}$ tuvieron una variación significativamente mayor que a 24 y $28^{\circ} \mathrm{C}(\mathrm{H}: \mathrm{W}=$ $10,351 ; \mathrm{p}<0,0001$ y $\mathrm{M}: \mathrm{W}=11,291 ; \mathrm{p}<0,0001)$.

En el ANDEVA realizado para evaluar los efectos producidos por la temperatura, la procedencia de las progenies analizadas (familias) y el sexo sobre la variación en la longitud del ala derecha (Tabla 4) se observó que la temperatura $(\mathrm{F}=$
$338,6603 ; \mathrm{p}<0,0001)$ y la familia $(\mathrm{F}=16,1903 ; \mathrm{p}<0,0001)$ tienen efectos significativos mientras que el sexo no $(\mathrm{F}=$ $1,4213 ; \mathrm{p}=0,2356)$. Al descomponer la variación fenotípica de la longitud del ala derecha, el factor temperatura, hizo el mayor aporte a la variación $(96,5 \%)$, seguido del factor familia con 2,9\% (Tabla 4).

La tasa largo/ancho de las alas derechas fue variable entre las progenies de las diferentes familias y se mostró como una característica importante para describir el alargamiento del ala. Los análisis descriptivos se presentan en la Tabla 5. Con excepción de la familia tres, la tasa media del largo/ancho de las alas derechas fue mayor en machos que en hembras. Hay un claro dimorfismo sexual para el alargamiento promedio del ala. El alargamiento promedio en las progenies hembras fue significativamente menor que la de las progenies machos tanto en las familias 2, 6-9 (Tabla 6) como cuando se consideraron todas las progenies $(\mathrm{t}$-student $=-5,1364 ; \mathrm{p}<0,0001)$.

El alargamiento promedio en hembras fue 3,575 $\pm 0,232$ mientras que en machos fue 3,798 $\pm 0,2010$. Este dimorfismo, también se mantuvo considerando cada temperatura de cría de las larvas. Los valores de t-student fueron: - 3,0810 a $20{ }^{\circ} \mathrm{C}$ $(\mathrm{p}=0,0041),-5,0455$ a $24^{\circ} \mathrm{C}(\mathrm{p}<0,0001)$ y $-2,7638$ a $28^{\circ} \mathrm{C}$ $(\mathrm{p}=0,0098)$. Estos resultados sugieren que las alas de los machos son significativamente menos anchas que las de las hembras (Tablas 6 y 7). También se puede observar que el $70 \%$ de las tasas largo/ancho del ala derecha en hembras se registra entre 3,50 y 3,70 mientras que, en machos el 68,0\% se observa entre 3,9 y 4,1 (Fig. 3).

En el ANDEVA para evaluar los efectos producidos por la temperatura, la procedencia de las progenies (familias) y el sexo sobre la variación en la tasa largo/ancho del ala derecha (Tabla 4) se observó que la temperatura $(\mathrm{F}=616,7516$; $\mathrm{p}<0,0001)$, la familia $(\mathrm{F}=41,02 ; \mathrm{p}<0,0001)$ y el sexo $(\mathrm{F}=28,72 ; \mathrm{p}=0,0004)$ tienen efectos significativos. Al descomponer la variación fenotípica de la tasa largo/ancho del ala derecha, el factor temperatura hizo el mayor aporte a la variación $(98,0 \%)$, seguido del factor familia con $1,6 \%$

Tabla 2. Longitud de las alas derechas ( $\mathrm{mm}$ ) de Nyssorhynchus triannulatus criados en cámaras ambientales a tres temperaturas: $20^{\circ} \mathrm{C}$ (familias $1-4$ ), $24{ }^{\circ} \mathrm{C}$ (familias 5-8) y $28{ }^{\circ} \mathrm{C}$ (familias 9-11) considerando la procedencia de las progenies (familias) y el sexo.

\begin{tabular}{|c|c|c|c|c|c|c|c|c|c|c|c|c|}
\hline \multirow{3}{*}{ Sexo } & \multicolumn{12}{|c|}{ Longitud ala derecha $(\mathrm{mm})$} \\
\hline & \multirow{2}{*}{\begin{tabular}{|c|} 
Temp. \\
Familia
\end{tabular}} & \multicolumn{4}{|c|}{$20^{\circ} \mathrm{C}$} & \multicolumn{4}{|c|}{$24^{\circ} \mathrm{C}$} & \multicolumn{3}{|c|}{$28^{\circ} \mathrm{C}$} \\
\hline & & 1 & 2 & 3 & 4 & 5 & 6 & 7 & 8 & 9 & 10 & 11 \\
\hline \multirow{5}{*}{ Hembra } & $\mathrm{N}$ & 8 & 7 & 7 & 6 & 10 & 6 & 10 & 12 & 11 & 10 & 7 \\
\hline & Media & 2,626 & 2,355 & 3,043 & 3,162 & 2,986 & 2,562 & 2,582 & 2,767 & 2,609 & 2,522 & 2,830 \\
\hline & D.E. & 0,399 & 0,119 & 0,094 & 0,051 & 0,210 & 0,239 & 0,153 & 0,232 & 0,274 & 0,251 & 0,284 \\
\hline & Mínimo & 2,200 & 2,130 & 2,880 & 3,080 & 2,600 & 2,300 & 2,370 & 2,360 & 2,340 & 2,220 & 2,440 \\
\hline & Máximo & 3,300 & 2,500 & 3,160 & 3,220 & 3,220 & 2,900 & 2,880 & 3,200 & 3,150 & 2,910 & 3,170 \\
\hline \multirow{5}{*}{ Macho } & $\mathrm{N}$ & 4 & 4 & 3 & 3 & 7 & 6 & 5 & 3 & 3 & 6 & 3 \\
\hline & Media & 2,553 & 2,290 & 2,983 & 3,210 & 2,813 & 2,478 & 2,672 & 2,713 & 2,750 & 2,548 & 2,393 \\
\hline & D.E. & 0,312 & 0,058 & 0,129 & 0,275 & 0,177 & 0,116 & 0,224 & 0,055 & 0,132 & 0,237 & 0,185 \\
\hline & Mínimo & 2,310 & 2,240 & 2,890 & 2,910 & 2,580 & 2,400 & 2,430 & 2,650 & 2,650 & 2,260 & 2,210 \\
\hline & Máximo & 3,000 & 2,340 & 3,130 & 3,450 & 3,030 & 2,710 & 3,020 & 2,750 & 2,900 & 2,970 & 2,580 \\
\hline
\end{tabular}

D. E.: Desviación estándar; Temp.: Temperatura. 
Tabla 3. Pruebas de t-student con su significancia (p) para comparar longitud promedio de progenies hembras $(\mathrm{H})$ y machos $(\mathrm{M})$ en cada familia de Nyssorhynchus triannulatus criadas a $20^{\circ} \mathrm{C}, 24^{\circ} \mathrm{C}$ y $28^{\circ} \mathrm{C}$.

\begin{tabular}{cccccc}
\hline Temperatura $\left({ }^{\circ} \mathbf{C}\right)$ & Familia No. & $\mathbf{N}\left(\boldsymbol{n}_{\boldsymbol{1}}+\boldsymbol{n}_{\mathbf{2}}\right)-\mathbf{2}$ & $\overline{\boldsymbol{X}}_{\boldsymbol{H}}-\overline{\boldsymbol{X}}_{\boldsymbol{M}}$ & $\mathbf{t}$ & $\mathbf{p}$ \\
\hline \multirow{2}{*}{20} & 1 & 7 & 0,5700 & 13,80 & $<0,0001$ \\
& 2 & 7 & 0,6110 & 35,24 & $<0,0001$ \\
& 3 & 10 & 0,1081 & 9,14 & $<0,0001$ \\
& 4 & 9 & 0,0300 & 2,43 & 0,0453 \\
\hline \multirow{2}{*}{24} & 5 & 16 & 0,1440 & 13,841 & $<0,0001$ \\
& 6 & 13 & $-0,1371$ & $-6,354$ & 0,0001 \\
& 7 & 9 & $-0,1480$ & $-7,024$ & 0,0002 \\
& 8 & 13 & $-0,0323$ & $-1,228$ & 0,2451 \\
\hline \multirow{2}{*}{28} & 9 & 11 & 0,0400 & 1,084 & 0,3200 \\
& 10 & 9 & $-0,0162$ & $-0,741$ & 0,4775 \\
& 11 & 0,4111 & 11,951 & $<0,0001$ \\
\hline
\end{tabular}

$n_{l}$ : Número de hembras por familia; $n_{2}$. Número de machos por familia. $\bar{X}_{H}$ : Tasa largo/ancho promedio en hembras. $\bar{X}_{M}$ : Tasa largo/ancho promedio en machos.

Tabla 4. Componentes de la variación fenotípica (\%), prueba F para contrastar variación y su significancia (p), para los caracteres longitud, tamaños de manchas costales, de la fusión (SP + PRSD + ASP) y las proporciones del ala derecha de progenies hembras y machos de Nyssorhynchus triannulatus, considerando como factores las temperaturas $(\mathrm{T})$ de crianza $\left(20,24\right.$ y $\left.28{ }^{\circ} \mathrm{C}\right)$, las procedencias $($ familias $=\mathrm{F})$ y sexo $(\mathrm{S})$ con las interacciones significativas (Temperatura*Sexo $=\mathrm{T} * \mathrm{~S}$ y Familia*Sexo $=\mathrm{F} * \mathrm{~S})$.

\begin{tabular}{|c|c|c|c|c|c|c|c|c|c|c|c|c|c|c|c|c|c|}
\hline \multirow{2}{*}{ Caracteres } & \multicolumn{6}{|c|}{ Componentes de variación (\%) } & \multirow{2}{*}{$\begin{array}{c}\text { Variación } \\
\text { Total }\end{array}$} & \multicolumn{2}{|c|}{ Temperatura } & \multicolumn{2}{|c|}{ Familia } & \multicolumn{2}{|c|}{ Sexo } & \multicolumn{2}{|c|}{$T * S$} & \multicolumn{2}{|c|}{$F^{*} S$} \\
\hline & T. & $\begin{array}{l}\text { Familia } \\
\text { (F) }\end{array}$ & $\begin{array}{l}\text { Sexo } \\
\text { (S.) }\end{array}$ & $\mathbf{T} * \mathbf{S}$ & $F^{*} \mathrm{~S}$ & Error & & $\mathbf{F}$ & $\mathbf{p}$ & $\mathbf{F}$ & $\mathbf{p}$ & $\mathbf{F}$ & $\mathbf{p}$ & $\mathbf{F}$ & $\mathbf{p}$ & $\mathbf{F}$ & $\mathbf{p}$ \\
\hline Longitud (mm) & $96,5 \%$ & $2,9 \%$ & & & & $0,6 \%$ & 7,41030 & 338,66 & $<0,0001$ & 71,88 & $<0,0001$ & 1,68 & 0,2288 & & & 0,86 & 0,5759 \\
\hline Largo/Ancho & $98,0 \%$ & $1,6 \%$ & $0,1 \%$ & & & $0,2 \%$ & 13,49410 & 616,75 & $<0,0001$ & 41,02 & $<0,0001$ & 28,72 & 0,0004 & & & 1,75 & 0,0808 \\
\hline $\mathrm{BP}+\mathrm{PHP}(\mathrm{mm})$ & & $3,2 \%$ & & & & $96,8 \%$ & 0,001421 & & & 1,21 & 0,3197 & & & & & & \\
\hline PHD (mm) & $3,3 \%$ & $41,9 \%$ & & & & $54,9 \%$ & 0,002077 & 9,58 & 0,0002 & 7,49 & $<0,0001$ & & & & & & \\
\hline $\mathrm{HP}(\mathrm{mm})$ & $6,3 \%$ & $12,3 \%$ & $0,7 \%$ & & & $80,7 \%$ & 0,000731 & 3,71 & 0,0337 & 2,44 & 0,0269 & 1,42 & 0,2479 & & & & \\
\hline $\mathrm{HD}(\mathrm{mm})$ & $6,2 \%$ & $43,8 \%$ & $0,8 \%$ & & & $49,2 \%$ & 0,001743 & 5,39 & 0,0071 & 10,04 & $<0,0001$ & 1,77 & 0,1932 & & & & \\
\hline PSP (mm) & & $26,0 \%$ & & & & $74,0 \%$ & 0,001110 & & & 4,42 & $<0,0001$ & & & & & & \\
\hline PSD (mm) & $0,8 \%$ & $21,2 \%$ & $7,1 \%$ & & & $70,9 \%$ & 0,002341 & 1,37 & 0,2821 & 3,75 & 0,0015 & 5,80 & 0,0222 & & & & \\
\hline $\mathrm{SP}(\mathrm{mm})$ & & $10,8 \%$ & $0,5 \%$ & & & $88,7 \%$ & 0,001259 & & & 2,11 & 0,0323 & 1,28 & 0,2615 & & & & \\
\hline PRSD (mm) & $2,8 \%$ & $16,0 \%$ & & & & $81,2 \%$ & 0,000926 & 4,09 & 0,0198 & 2,89 & 0,0063 & & & & & & \\
\hline $\operatorname{ASP}(\mathrm{mm})$ & $3,7 \%$ & $18,9 \%$ & & & & $77,4 \%$ & 0,002174 & 2,61 & 0,0791 & 3,16 & 0,0032 & & & & & & \\
\hline $\mathrm{DSD}(\mathrm{mm})$ & $11,0 \%$ & $21,7 \%$ & $3,1 \%$ & $4,2 \%$ & & $60,1 \%$ & 0,003031 & 8,18 & 0,0006 & 4,32 & 0,0002 & 4,45 & 0,0396 & 1,95 & 0,1545 & & \\
\hline $\mathrm{SCP}(\mathrm{mm})$ & $1,6 \%$ & $26,7 \%$ & $\begin{array}{l}10,5 \\
\%\end{array}$ & & & $61,2 \%$ & 0,001433 & 1,91 & 0,1705 & 5,08 & 0,0001 & 9,48 & 0,0036 & & & & \\
\hline $\mathrm{PD}(\mathrm{mm})$ & $0,8 \%$ & $7,6 \%$ & $5,7 \%$ & $3,6 \%$ & & $82,3 \%$ & 0,003295 & 1,88 & 0,1592 & 1,81 & 0,0867 & 4,75 & 0,0321 & 1,55 & 0,2188 & & \\
\hline $\mathrm{PP}(\mathrm{mm})$ & $2,8 \%$ & $1,7 \%$ & $4,4 \%$ & $5,8 \%$ & $8,2 \%$ & $77,1 \%$ & 0,000963 & 4,20 & 0,0188 & 1,75 & 0,1014 & 4,68 & 0,0338 & 2,27 & 0,1110 & 1,37 & 0,2230 \\
\hline $\mathrm{AD}(\mathrm{mm})$ & $2,4 \%$ & $32,6 \%$ & & & & $65,0 \%$ & 0,001416 & 5,71 & 0,0050 & 4,65 & 0,0001 & & & & & & \\
\hline $\mathrm{AP}(\mathrm{mm})$ & & $22,4 \%$ & & & & $77,6 \%$ & 0,001054 & & & 3,22 & 0,0017 & & & & & & \\
\hline HP/PHD & $12,0 \%$ & $38,1 \%$ & & & & $49,9 \%$ & 0,140186 & 14,53 & $<0,0001$ & 7,15 & $<0,0001$ & & & & & & \\
\hline SCP/DSD & $8,4 \%$ & $42,5 \%$ & $8,0 \%$ & $5,3 \%$ & & $35,8 \%$ & 0,008796 & 11,03 & 0,0001 & 11,95 & $<0,0001$ & 13,95 & 0,0004 & 3,01 & 0,0570 & & \\
\hline $\begin{array}{l}\mathrm{SP}+\mathrm{PRSD}+ \\
\mathrm{ASP}\end{array}$ & $69,4 \%$ & $11,4 \%$ & & & & $19,2 \%$ & 0,009132 & 133,60 & $<0,0001$ & 6,84 & $<0,0001$ & & & & & & \\
\hline $\mathrm{PB} / \mathrm{ta}^{-\mathrm{III}}{ }_{2}$ & $25,5 \%$ & $45,3 \%$ & & & & $29,2 \%$ & 0,003723 & 38,28 & $<0,0001$ & 13,18 & $<0,0001$ & & & & & & \\
\hline
\end{tabular}


Tabla 5. Tasa largo/ancho de las alas derechas de Nyssorhynchus triannulatus criadas en cámaras ambientales, a tres temperaturas: $20{ }^{\circ} \mathrm{C}$ (familias $1-4), 24{ }^{\circ} \mathrm{C}$ (familias 5-8) y $28^{\circ} \mathrm{C}$ (familias 9-11) considerando la procedencia de las progenies (familias) y el sexo.

Tasa largo/ancho del ala derecha

\begin{tabular}{|c|c|c|c|c|c|c|c|c|c|c|c|c|}
\hline \multirow{2}{*}{ Sexo } & \multirow{2}{*}{$\begin{array}{c}\text { Temp. } \\
\text { Familia }\end{array}$} & \multicolumn{4}{|c|}{$20^{\circ} \mathrm{C}$} & \multicolumn{4}{|c|}{$24^{\circ} \mathrm{C}$} & \multicolumn{3}{|c|}{$28^{\circ} \mathrm{C}$} \\
\hline & & 1 & 2 & 3 & 4 & 5 & 6 & 7 & 8 & 9 & 10 & 11 \\
\hline \multirow{5}{*}{ Hembra } & $\mathrm{N}$ & 5 & 6 & 7 & 4 & 8 & 6 & 8 & 12 & 10 & 8 & 5 \\
\hline & Media & 3,518 & 3,584 & 3,568 & 3,510 & 3,653 & 3,406 & 3,523 & 3,627 & 3,529 & 3,690 & 3,678 \\
\hline & D.E. & 0,055 & 0,146 & 0,060 & 0,114 & 0,181 & 0,081 & 0,271 & 0,105 & 0,099 & 0,285 & 0,139 \\
\hline & Mínimo & 3,420 & 3,430 & 3,490 & 3,390 & 3,430 & 3,320 & 3,250 & 3,470 & 3,360 & 3,330 & 3,490 \\
\hline & Máximo & 3,560 & 3,860 & 3,650 & 3,640 & 4,000 & 3,540 & 4,060 & 3,820 & 3,650 & 4,070 & 3,770 \\
\hline \multirow{5}{*}{ Macho } & $\mathrm{N}$ & 4 & 4 & 3 & 3 & 7 & 5 & 5 & 2 & 3 & 3 & 2 \\
\hline & Media & 3,735 & 4,005 & 3,543 & 3,523 & 3,809 & 3,842 & 3,834 & 3,915 & 3,793 & 3,913 & 3,775 \\
\hline & D.E. & 0,236 & 0,070 & 0,101 & 0,061 & 0,183 & 0,235 & 0,173 & 0,049 & 0,355 & 0,049 & 0,247 \\
\hline & Mínimo & 3,480 & 3,900 & 3,480 & 3,470 & 3,540 & 3,450 & 3,590 & 3,880 & 3,410 & 3,880 & 3,600 \\
\hline & Máximo & 3,950 & 4,050 & 3,660 & 3,590 & 4,070 & 4,080 & 4,030 & 3,950 & 4,110 & 3,970 & 3,950 \\
\hline
\end{tabular}

D. E.: Desviación estándar; Temp.: Temperatura.

y por último el sexo con $0,1 \%$ (Tabla 4 ). Al igual que la variación en la longitud total del ala derecha, la tasa largo/ ancho fue susceptible a cambios ambientales, en este caso, a la temperatura de cría de las progenies. Igualmente, la $\mathrm{H}^{2}$ estimada en sentido amplio fue baja $(2 * 1,6 \%=3,2 \%)$. Esto significa que este carácter proporcionalmente tiene un componente genético bajo, lo cual posiblemente se deba a que los individuos dentro de las familias fueron muy variables $\mathrm{y}$, por ende, el residual se hace proporcionalmente mayor respecto al componente entre familias de individuos criados a la misma temperatura (Tabla 4).

En el Anexo 1 se indican los promedios con sus respectivas desviaciones estándar (D. E.) de la longitud de las alas derechas, de los tamaños de las manchas costales, de las proporciones promedio y de la tasa de la mancha oscura basal/ longitud del tarsómero posterior dos ( $\left.\mathrm{PB} / \mathrm{ta}^{-} \mathrm{III}_{2}\right)$ en progenies hembras y machos de Nys. triannulatus, criadas a tres temperaturas: 20,24 y $28^{\circ} \mathrm{C}$. Con base en el Anexo 1 se confirmó la existencia de dimorfismo sexual en cada una de las temperaturas de crianza (Tabla 5). Entre el 80 y $85 \%$ de los caracteres mostraron diferencias significativas entre sexos. En cualquiera de las tres temperaturas de crianza, la longitud promedio del ala es significativamente mayor en hembras respecto a machos, mientras que los promedios de los tamaños de SCP y PP y de la tasa SCP/DSD fueron menores en hembras respecto a machos. A $20{ }^{\circ} \mathrm{C}$, los promedios de la longitud de las manchas BP + PHP, SP, PRSD, de la fusión (SP + PRSD + ASP) y de la tasa HP/PHD fueron significativamente mayores en hembras respecto a machos, mientras que las manchas PHD, HP, HD, PSD, ASP, SCP, PD, PP, AD y AP, las tasas SCP/DSD y PB/ta-III 2 fueron significativamente mayores en machos respecto a hembras.

A $24{ }^{\circ} \mathrm{C}$, los promedios de la longitud de las manchas BP + PHP, PSP, PRSD, DSD, PD, AD y AP, la tasa HP/PHD y de la tasa $\mathrm{PB} / \mathrm{ta}-\mathrm{III}_{2}$ fueron significativamente mayores en hembras respecto a machos, mientras que las manchas PHD, HP, HD, PSD, SP, SCP y PP, la tasa SCP/DSD y la longitud de la fusión (SP + PRSD + ASP) mostró lo contrario. A 28
${ }^{\circ} \mathrm{C}$, los promedios de la longitud de la mancha $\mathrm{PD}$, de la tasa HP/PHD fueron significativamente mayores en hembras respecto a machos, mientras que las manchas BP + PHP, PHD, HP, HD, PSP, SP, ASP, SCP, PP y AP, las tasas SCP/DSD y PB/ta-III ${ }_{2}$ la fusión (SP + PRSD + ASP) en los machos fueron significativamente mayores respecto a hembras. Al parecer, a esta temperatura de cría, en machos se favorece el aumento de la longitud de las manchas claras sobre las manchas oscuras.

Los tamaños promedio de las manchas costales PRSD y DSD fueron mayores en hembras respecto a machos a cualquiera de las tres temperaturas de crianza, pero solo significativas a $24^{\circ} \mathrm{C}$. La tasa HP/PHD fue mayor en hembras respecto a machos, pero esta diferencia no fue significativa a $24^{\circ} \mathrm{C}$. Los tamaños promedio de PHD, HP y HD fueron menores en hembras respecto a machos a cualquiera de las tres temperaturas de crianza, pero no fue significativo para $\mathrm{HP}$ a $24{ }^{\circ} \mathrm{C}$. El tamaño promedio de la mancha PSD fue menor en hembras respecto a machos, pero esta diferencia no fue significativa a $28^{\circ} \mathrm{C}$. El tamaño promedio de la mancha $\mathrm{BP}+\mathrm{PHP}$ inicialmente significativamente mayor en hembras respecto a machos a $20^{\circ} \mathrm{C}$ y $24{ }^{\circ} \mathrm{C}$, se hace significativamente menor a $28{ }^{\circ} \mathrm{C}$. También muestra esta tendencia PSP. El tamaño promedio de la mancha SP que a $20^{\circ} \mathrm{C}$ es significativamente mayor en hembras respecto a machos, se hace significativamente menor a $24{ }^{\circ} \mathrm{C}$ y $28{ }^{\circ} \mathrm{C}$, triplicándose esta diferencia a $28^{\circ} \mathrm{C}$.

La fusión (SP + PRSD + ASP) también muestra esta tendencia. El tamaño promedio de la mancha $\mathrm{AD}$ que a $20^{\circ} \mathrm{C}$ es significativamente menor en hembras respecto a machos, se hace significativamente mayor a $24^{\circ} \mathrm{C}$ y $28^{\circ} \mathrm{C}$. El tamaño promedio de la mancha $\mathrm{PD}$ es significativamente menor en hembras respecto a machos a $20^{\circ} \mathrm{C}$, mayor a $24^{\circ} \mathrm{C}$ y vuelve a ser significativamente menor a $28{ }^{\circ} \mathrm{C}$. También muestra esta tendencia la tasa $\mathrm{PB} / \mathrm{ta}-\mathrm{III}_{2}$.

Las pruebas estadísticas por cada una de las temperaturas de crianza por familia se muestran en el Anexo 2. Entre el 68 y el $95 \%$ de la longitud promedio de los caracteres analiza- 
Tabla 6. Pruebas de t-student con su significancia (p) para comparar la tasa promedio largo/ancho del ala derecha de progenies hembras y machos en cada familia de Nyssorhynchus triannulatus criadas a $20,24,28^{\circ} \mathrm{C}$.

\begin{tabular}{cccccc}
\hline Temperatura $\left({ }^{\circ} \mathbf{C}\right)$ & Familia No. & $\mathbf{N}\left(\boldsymbol{n}_{1}+\boldsymbol{n}_{2}\right)-\mathbf{2}$ & $\overline{\boldsymbol{X}}_{\boldsymbol{H}}-\overline{\boldsymbol{X}}_{\boldsymbol{M}}$ & $\mathbf{t}$ & $\mathbf{p}$ \\
\hline & 1 & 7 & $-0,2175$ & $-2,0300$ & 0,0819 \\
20 & 2 & 8 & $-0,4210$ & $-5,2923$ & 0,0007 \\
& 3 & 8 & 0,0250 & 0,4996 & 0,6308 \\
& 4 & 5 & $-0,0133$ & $-0,1813$ & 0,8633 \\
\hline & 5 & 13 & $-0,1557$ & $-1,6557$ & 0,1217 \\
& 6 & 9 & $-0,4360$ & $-4,2972$ & 0,0020 \\
& 7 & 11 & $-0,3111$ & $-2,2728$ & 0,0441 \\
& 8 & 12 & $-0,2877$ & $-3,7068$ & 0,0030 \\
\hline & 9 & 11 & $-0,2644$ & $-2,2878$ & 0,0429 \\
& 10 & 9 & $-0,2233$ & $-1,3090$ & 0,2230 \\
& 11 & 5 & $-0,0975$ & $-0,7013$ & 0,5144 \\
\hline
\end{tabular}

$n_{1}$ : Número de hembras por familia; $n_{2}$ : Número de machos por familia; $\bar{X}_{H}$ : Tasa largo/ancho promedio en hembras; $\bar{X}_{M}$ : Tasa largo/ancho promedio en machos.

dos mostraron diferencias significativas entre las progenies hembras y machos. De estas diferencias, entre el 16 y el 60 $\%$ mostraron longitudes promedio de las hembras mayores que las de los machos y del 40 al $84 \%$ de las comparaciones mostraron lo contrario. De las 11 familias analizadas, siete mostraron la longitud promedio de las alas de hembras significativamente mayor que las de los machos $(7 / 11=0,636)$, mientras que las manchas HP $(10 / 11=0,909)$, PSD $(8 / 11$ $=0,727), \mathrm{SCP}(9 / 11=0,818), \mathrm{PP}(7 / 11=0,636)$ mostraron longitudes promedio de las manchas costales de los machos

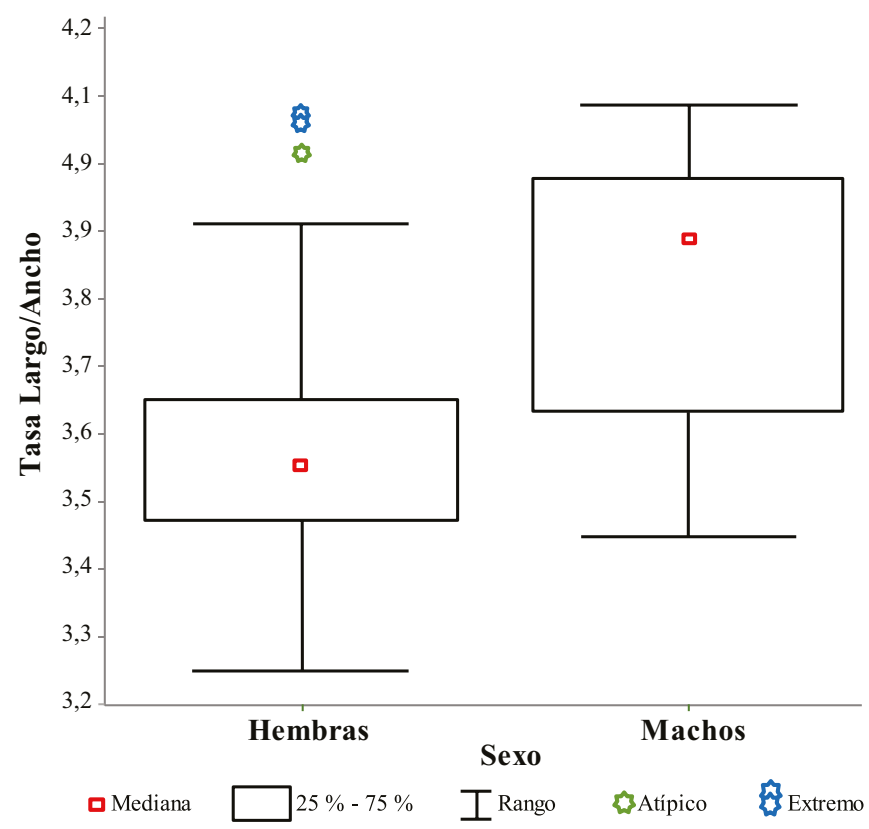

Figura 3. Diagrama de caja mostrando la distribución de las tasas largo/ancho de las alas derechas de hembras y machos de Nyssorhynchus triannulatus. En resumen, se indican cinco números: "mínimo", primer cuartil (Q1), mediana, tercer cuartil (Q3) y "máximo". También se indican los valores atípicos observados en hembras (4 entre 4,0 y 4,1). significativamente mayores que las de las hembras. La tasa promedio de SCP/DSD en las progenies machos fue significativamente mayor que las de las hembras en 7 de las 11 familias $(7 / 11=0,636)$. Esto se debe principalmente a que la longitud promedio de la mancha costal SCP es significativamente mayor que la de las hembras en 9 de 11 familias analizadas.

En las progenies criadas a $20^{\circ} \mathrm{C}$, la relación de las longitudes promedio de DSD y SCP varia, por ejemplo, en las familias 1 y 2 la diferencia entre sexos (H-M) es positiva para DSD y negativa para SCP mientras que en las familias 3 y 4 ambas son negativas y en la familia 4 no se observa dimorfismo sexual en estas manchas. En cambio, en las progenies criadas a $24^{\circ} \mathrm{C}$ la diferencia entre sexos $(\mathrm{H}-\mathrm{M})$ es positiva para DSD y negativa para SCP en las 4 familias estudiadas y son significativas para el dimorfismo sexual. En las progenies criadas a $28^{\circ} \mathrm{C}$, en la familia 9 , la diferencia entre sexos es negativa y significativa para DSD y positiva no significativa para SCP; en la familia 10 es positiva para DSD y negativa para SCP, ambas significativas; y en la familia 11 DSD y SCP son negativas y significativas para dimorfismo sexual. Esta variación en la dirección del dimorfismo sexual a temperaturas de $20{ }^{\circ} \mathrm{C}$ y $28{ }^{\circ} \mathrm{C}$ indica el efecto que la temperatura sobre las longitudes de las manchas de este sector. Parece ser que la temperatura de $24{ }^{\circ} \mathrm{C}$ es óptima debido a que muestra mayor estabilidad fenotípica (Tabla 7, Anexos 1 y 2 ).

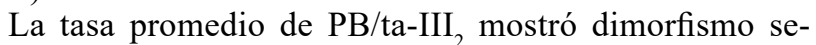
xual significativo en ocho de nueve familias $(8 / 9=0,889)$, en cinco fue significativamente mayor en hembras que en machos $(5 / 8=0,625)$ y en tres lo contrario $(3 / 8=0,375)$. Este dimorfismo sexual no se asocia significativamente con la temperatura de cría de las progenies $(-1,5156 \leq \mathrm{t} \leq$ $0,0188 ; 0,1397 \leq \mathrm{p} \leq 0,9885)$.

El mayor dimorfismo sexual se detectó en la familia 11 con 19 caracteres con diferencias significativas entre sexos $(19 / 20=0,95)$. De estas diferencias el $16 \%$ mostró longitudes promedio de hembras significativamente mayores que 
Tabla 7. Diferencias en las longitudes de alas, manchas y proporciones de manchas costales y tarsómero posterior dos, en progenies hembras y machos de Nyssorhynchus triannulatus, criados a tres temperaturas. Comparación de medias de hembras y machos dentro de cada temperatura $\left({ }^{\circ} \mathrm{C}\right)$ mediante Pruebas t-student y su significancia ( $\mathrm{p}<0,05 / 2=0,025$ para las proporciones).

\begin{tabular}{|c|c|c|c|c|c|c|c|c|c|}
\hline \multirow{2}{*}{ Característica } & \multicolumn{3}{|c|}{$20^{\circ} \mathrm{C}$} & \multicolumn{3}{|c|}{$24^{\circ} \mathrm{C}$} & \multicolumn{3}{|c|}{$28^{\circ} \mathrm{C}$} \\
\hline & $\begin{array}{c}\text { Diferencia } \\
\text { (H - M) }\end{array}$ & $\mathbf{t}$ & $\mathbf{p}$ & $\begin{array}{c}\text { Diferencia } \\
(\mathrm{H}-\mathrm{M})\end{array}$ & $\mathbf{t}$ & $\mathbf{p}$ & $\begin{array}{c}\text { Diferencia } \\
(\mathrm{H}-\mathrm{M})\end{array}$ & $\mathbf{t}$ & $\mathbf{p}$ \\
\hline Longitud (mm) & 0,0826 & 6,850 & $<0,0001$ & 0,0191 & 3,258 & 0,0020 & 0,1545 & 15,261 & $<0,0001$ \\
\hline $\mathrm{BP}+\mathrm{PHP}^{*}$ & 0,0083 & 5,120 & 0,0001 & 0,0087 & 6,497 & $<0,0001$ & $-0,0128$ & $-10,569$ & $<0,0001$ \\
\hline PHD* & $-0,0190$ & $-8,788$ & $<0,0001$ & $-0,0110$ & $-11,305$ & $<0,0001$ & $-0,0239$ & $-15,972$ & $<0,0001$ \\
\hline HP* & $-0,0139$ & $-10,690$ & $<0,0001$ & $-0,0002$ & $-0,403$ & 0,6891 & $-0,0062$ & $-7,514$ & $<0,0001$ \\
\hline $\mathrm{HD}^{*}$ & $-0,0058$ & $-3,998$ & 0,0004 & $-0,0143$ & $-19,583$ & $<0,0001$ & $-0,0073$ & $-6,762$ & $<0,0001$ \\
\hline PSP* & 0,0015 & 1,591 & 0,1224 & 0,0163 & 21,705 & $<0,0001$ & $-0,0219$ & $-26,246$ & $<0,0001$ \\
\hline PSD* & $-0,0240$ & $-16,674$ & $<0,0001$ & $-0,0309$ & $-34,941$ & $<0,0001$ & $-0,0014$ & $-0,950$ & 0,3506 \\
\hline $\mathrm{SP}^{*}$ & 0,0036 & 2,558 & 0,0158 & $-0,0016$ & $-3,126$ & 0,0030 & $-0,0394$ & $-32,160$ & $<0,0001$ \\
\hline PRSD* & 0,0006 & 0,698 & 0,4910 & 0,0022 & 3,511 & 0,0010 & 0,0005 & 0,543 & 0,5917 \\
\hline ASP* & $-0,0235$ & $-20,400$ & $<0,0001$ & 0,0012 & 1,087 & 0,2824 & $-0,0122$ & $-13,905$ & $<0,0001$ \\
\hline DSD* & 0,0024 & 1,758 & 0,0889 & 0,0388 & 37,833 & $<0,0001$ & 0,0001 & 0,045 & 0,9644 \\
\hline SCP* & $-0,0130$ & $-18,940$ & $<0,0001$ & $-0,0292$ & $-33,889$ & $<0,0001$ & $-0,0155$ & $-21,962$ & $<0,0001$ \\
\hline $\mathrm{PD}^{*}$ & $-0,0039$ & $-2,624$ & 0,0134 & 0,0382 & 32,333 & $<0,0001$ & 0,0397 & 26,287 & $<0,0001$ \\
\hline $\mathrm{PP}^{*}$ & $-0,0068$ & $-7,671$ & $<0,0001$ & $-0,0053$ & $-7,235$ & $<0,0001$ & $-0,0396$ & $-38,138$ & $<0,0001$ \\
\hline $\mathrm{AD}^{*}$ & $-0,0074$ & $-5,171$ & $<0,0001$ & 0,0177 & 19,672 & $<0,0001$ & 0,0002 & 0,089 & 0,9300 \\
\hline $\mathrm{AP}^{*}$ & $-0,0174$ & $-15,218$ & $<0,0001$ & 0,0142 & 17,573 & $<0,0001$ & $-0,0328$ & $-23,082$ & $<0,0001$ \\
\hline $\mathrm{HP} / \mathrm{PHD}^{* *}$ & 0,0761 & 5,109 & $<0,0001$ & 0,0056 & 0,608 & 0,5473 & 0,1038 & 11,168 & $<0,0001$ \\
\hline $\mathrm{SCP} / \mathrm{DSD}^{* *}$ & $-0,0177$ & $-9,859$ & $<0,0001$ & $-0,0788$ & $-41,173$ & $<0,0001$ & $-0,0110$ & $-8,959$ & $<0,0001$ \\
\hline$(\mathrm{SP}+\mathrm{PRSD}+\mathrm{ASP})^{*}$ & 0,0134 & 8,134 & $<0,0001$ & $-0,0166$ & $-15,223$ & $<0,0001$ & $-0,0130$ & $-10,993$ & $<0,0001$ \\
\hline $\mathrm{PB} / \mathrm{ta}_{\mathrm{III}}{ }_{2} *$ & $-0,0039$ & $-2,265$ & 0,0315 & 0,0035 & 2,820 & 0,0075 & $-0,0328$ & $-11,216$ & $<0,0001$ \\
\hline
\end{tabular}

E.E.(p).: Error estándar del promedio (hembras + machos); (*) A partir de datos transformados según Freeman y Tukey (1950) y Kihlberg et al. (1972).

la de los machos y el $84 \%$ restante lo contrario. El menor dimorfismo sexual se detectó en la familia 7 con 12 caracteres con diferencias significativas entre sexos $(12 / 20=0,60)$. De estas diferencias el $42 \%$ mostró longitudes promedio de hembras significativamente mayores que la de los machos y el $58 \%$ restante reveló lo contrario. La familia 6 también mostró un dimorfismo significativo alto $(18 / 20=0,90)$ pero al contrario de la familia 11 , las longitudes promedio de hembras fueron significativamente mayores que la de los machos $(11 / 18=0,61)$ mientras que lo contrario de observó en el $39 \%$ restante. En general, existe dimorfismo sexual en los promedios de la longitud, el alargamiento, los tamaños de las manchas costales y en la tasa promedio $\mathrm{PB} / \mathrm{ta}_{\mathrm{III}}$. En todos los casos, se presentó variación en el dimorfismo sexual asociada con la procedencia (familias) de las progenies. En el 29,2\% de los casos con dimorfismo sexual se favorece la mayor longitud de las manchas oscuras en hembras mientras que en el $70,8 \%$ se favorece la mayor longitud de las manchas claras en machos.

En la Tabla 4 se registraron los componentes de la variación fenotípica (\%) aportados por los factores temperatura, familias y sexos con sus interacciones. Esta tabla muestra un efecto altamente significativo de la temperatura de crianza sobre la variación de la longitud, el alargamiento (tasa longitud/ancho) y la fusión de las manchas costales SP + PRSD + ASP del ala derecha, con una variación explicada alta, entre
$69,4 \%$ y $98,0 \%$; a las proporciones $\mathrm{PB} / \mathrm{ta}-\mathrm{III}_{2}, \mathrm{HP} / \mathrm{PHD}$ y SCP/DSD y DSD, con una variación explicada entre $8,4 \%$ y $25,5 \%$, a las machas HP, HD, PHD, PRSD, PP y AD con aportes a la variación fenotípica bajos, entre $2,4 \%$ y $6,3 \%$. No mostró un efecto significativo sobre la variación fenotípica de las manchas costales BP + PHP, PSP, PSD, SP, ASP, SCP, PD y AP.

En esta tabla también se observó un efecto altamente significativo de la familia sobre la variación fenotípica de las proporciones HP/PHD, SCP/DSD y $\mathrm{PB} / \mathrm{ta}^{-\mathrm{III}_{2}}$, de los tamaños de las manchas PHD, HD, PSP, PSD, DSD, SCP, AD y AP con una variación fenotípica explicada alta, entre $21,7 \%$ y 45,3 \%; de las manchas HP, SP, PRSD, y la fusión SP + PRSD + ASP, con aportes a la variación fenotípica entre $18,8 \%$ y $16,0 \%$. Además, mostró efectos significativos $(\mathrm{p}<0,05)$ pero aportes a la variación fenotípica bajos para la longitud $(2,9 \%)$ y la tasa largo/ancho $(1,6 \%)$.

El efecto del sexo sobre la variación fenotípica de los caracteres estudiados fue significativo para las tasas largo/ancho $(0,1 \%)$ y SCP/DSD $(8,0 \%)$ y los tamaños de las manchas PSD $(7,1 \%)$, DSD $(3,1 \%)$, SCP $(10,5 \%)$, PD $(5,7 \%)$ y PP $(4,4 \%)$. No mostró efecto significativo sobre la variación fenotípica de las manchas costales BP + PHP, PD y PP.

No se observaron interacciones significativas entre los tres factores analizados y los valores registrados en la Tabla 4 fueron menores al 8,2 \% (Familia*Sexo) para la mancha PP. 


\section{Discusión}

Pocas veces se presentó fusión de algunas de las manchas costales o por lo menos ausencia de la accesoria clara (ASP), lo cual corresponde a lo comúnmente observado en especímenes criados en su ambiente natural aunque, según Faran (1980), Nys. triannulatus s.l. presenta frecuentemente la vena C casi completamente oscura y las manchas claras ocasionalmente poco diferenciadas. Pero esto no parece estar de acuerdo con lo descrito formalmente para las especies de este complejo, dado que las manchas claras, a pesar de su tamaño pequeño, casi siempre están presentes. En estudios para esta misma especie, se ha observado, aunque en baja frecuencia, de tres a cuatro tipos de patrones costales adicionales, pero restringidos a progenies de familias obtenidas de muestras de población del departamento del Meta (Marín Londoño 2014).

Por otro lado, en la descripción original de Nys. halophylus, se indicó que ASP no está presente en el $30 \%$ de los ejemplares examinados, es decir que el patrón costal I podría estar cercano al $70 \%$, lo cual parece ser también frecuente en algunas poblaciones de Nys. triannulatus s.s. (Silva do Nascimento y Lourenço-de-Oliveira 2002). En algunas familias de muestras de poblaciones (datos no publicados) de los departamentos colombianos de Huila, Meta, Putumayo y Valle del Cauca, la frecuencia de este patrón varió entre 33,5 y $100 \%$, es decir que en esta especie este carácter es altamente polimórfico y parece contribuir a la plasticidad de los caracteres fenotípicos. El patrón costal II se puede confundir con el III (como se describe en el presente escrito), debido a que ambos involucran a la mancha costal ASP. Sin embargo, en el patrón II se expresa un tamaño-mayor de la mancha clara que involucra la SP, PRSD y ASP y por lo tanto, la SP es observada con mayor tamaño que en el patrón I, mientras que en el patrón III, hay expresión de la ASP, es decir que queda incorporada a la SD y por lo tanto, esta mancha oscura se observará más grande que en el patrón I.

Para mayor claridad, sobre los patrones costales II y III, conviene resaltar que para las especies del subgénero Nyssorhynchus, Wilkerson y Peyton (1990) definen al sector claro (SP) como un grupo de escamas claras presentes sobre la costa, subcostal y/o radio, que coinciden con la posición de la vena transversal sc-r, mientras que para la posición de la mancha costal ASP coincide la posición de la vena transversal $\mathrm{r}_{1}$-rs.

La temperatura en la cual ocurrió el desarrollo de las formas inmaduras, como en todos los organismos ectotermos, no solo influye de manera marcada en su velocidad de desarrollo preimagal (datos no evaluados) sino que también parece ser uno de los factores que contribuye a la variación en la expresión de patrones de manchas costales (Fig. 1). La relación entre las frecuencias de los patrones costales y el incremento de la temperatura $\left(20,24\right.$ y $\left.28^{\circ} \mathrm{C}\right)$ mostró asociación significativa en hembras $\left(\chi^{2}=7,4 ; p=0,0065\right)$ mientras que en machos no fue evidente $\left(\chi^{2}=0,03 ; p=0,8607\right)$. De acuerdo con esto, el incremento en la temperatura parece favorecer la aparición de fusiones de manchas costales en hembras, mientras que en machos el patrón de manchas parece ser más estable.

La longitud del ala es una característica altamente correlacionada con el tamaño del insecto, es decir que, a mayor tamaño de alas mayor tamaño corporal, por eso es considerado para especies de Culicidae como un estimador indirecto del tamaño corporal (Dujardin 2011). En las descripciones de las especies del complejo se ha observado que esta especie pre- senta un tamaño relativamente pequeño en comparación con otras especies del subgénero Nyssorhynchus (Faran 1980). En el presente estudio, la longitud promedio de las alas derechas en Nys. triannulatus fue significativamente menor que las descritas por Silva do Nascimento y Lourenço-de-Oliveira (2002) para Nys. triannulatus y similares a los de Nys. halophylus. Estos autores observaron diferencias morfométricas significativas entre Nys. halophylus y Nys. triannulatus $(\mathrm{p}<$ $0,05)$. Sin embargo, también hay que considerar que las condiciones ambientales de los criaderos (temperatura, densidad, físico química del agua y cantidad y calidad del alimento) influyen en el tamaño larval y por ende en el tamaño de pupas y adulto (Lanciani y Le 1995). Esto es una posible explicación para entender por qué las progenies obtenidas en laboratorio suelen ser más pequeñas que las criadas en su ambiente natural.

Por otro lado, independiente de las características genéticas de cada especie y de la temperatura en la cual ocurre su desarrollo larval, se ha observado que la alimentación larval en especies de Anopheles afecta no solo el tamaño sino también la velocidad de su desarrollo, la supervivencia y caracteres biológicos de los adultos (Araújo et al. 2012), esto incluye, desde luego, su tamaño corporal y en general "fitness" de cada población o especie. Con base en los resultados del presente estudio (Tabla 2), la longitud del ala es una característica altamente variable entre las familias, aun para progenies criadas a la misma temperatura. Las diferencias en tamaño de alas, encontradas entre progenies obtenidas en temperaturas de 20 y $24{ }^{\circ} \mathrm{C}$, pueden explicar en parte las diferencias observadas entre las poblaciones de algunas especies de Anopheles (Rodríguez-Zabala et al. 2016; Pacheco et al. 2017; Pacheco-Gómez et al. 2018).

Es decir que en condiciones naturales es también posible que la longitud pueda ser un marcador morfológico de poblaciones y debido a que el tamaño corporal puede influenciar la capacidad vectorial de especies de Anopheles, es importante incluir la longitud alar como un estimador indirecto de su importancia epidemiológica para la malaria (verificable con datos de incriminación), taxonomía y asociación con aspectos propios de su bionomía (Pacheco-Gómez et al. 2018).

En anofelinos se ha observado que la reserva de energía que requiere una hembra para la búsqueda de su fuente de alimentación con sangre depende de su tamaño corporal (Dudley 2002). Un mayor tamaño puede estar relacionado con un mayor vigor y esto con una mayor longevidad. Esto es de gran importancia epidemiológica porque se relaciona con una mayor competencia vectorial para la transmisión del agente etiológico de la malaria, ya que realizan mayor número de ingestas de sangre, necesarias para el desarrollo del ciclo gonotrófico de cada hembra, y con esto se incrementa la probabilidad de adquirir y transmitir los parásitos de la malaria humana. Por otro lado, la forma y longitud de las alas constituyen características que contribuyen a definir la eficacia del vuelo (Dudley 2002), rasgo fundamental para la búsqueda de sitios aptos para la oviposición, fuentes de sangre (Charlwood 1996), la dispersión de genes de resistencia a insecticidas (Norberg 1995) y la propagación de esporozoitos de Plasmodium spp. (Dudley 2002). Estos rasgos del ala no dependen solo del acervo genético (Fusco y Minelli 2010) sino que son afectados por variables ambientales, como la temperatura, la humedad relativa y la altitud (Gómez et al. 2013; Gómez et al. 2014; Lorenz et al. 2014). 
La variación en la longitud del ala derecha fue muy susceptible a cambios de temperatura (Tabla 7). Si se asume que las familias de hermanos completos estiman el componente genético de la generación parental, la $\mathrm{H}^{2}$ estimada en sentido amplio fue baja $(2 * 2,9 \%=5,8 \%)$. Esto significa que este carácter tiene un componente genético proporcionalmente bajo, posiblemente atribuible a que los individuos dentro de las familias fueron muy variables y por ende el residual se hace proporcionalmente mayor respecto al componente entre familias de individuos criados a la misma temperatura. Estos resultados coinciden con los encontrados por Gómez y Correa (2017) quienes a partir del análisis morfométrico de $A n$. albimanus concluyen que el tamaño del ala es variable a nivel intra e interespecífico puesto que cada subgénero presenta una tendencia de tamaño de ala particular, posiblemente relacionada con la historia evolutiva de estos linajes.

Los resultados indicaron que las alas de esta especie tienden a ser estrechas y por ende presentan menor resistencia inducida (Cerón-Muñoz et al. 2013). Por otro lado, sugieren que las de los machos son significativamente menos anchas que las de las hembras. Desde el punto de vista adaptativo, un ala más ancha tiene más resistencia inducida y menos resistencia parasita que un ala alargada y más estrecha como la de los machos. Esto significa que las alas en hembras son más resistentes. Posiblemente esto esté asociado con el mayor tamaño o peso de las hembras mientras que la resistencia parasita es menor en machos, lo cual favorece una mayor dispersión al aumentar la velocidad de desplazamiento activo (Bomphrey et al. 2017).

Las dos variables, longitud y tasa largo/ancho mostraron un aporte muy alto del efecto temperatura. Sin embargo, este componente podría estar sobreestimado por el ambiente común dentro de cada uno de los tres niveles de temperatura $\left(20,24\right.$ y $\left.28^{\circ} \mathrm{C}\right)$ generando más parecido entre las familias del mismo nivel por el efecto de condiciones adicionales como la cantidad y calidad de la alimentación, aspectos fisicoquímicos del agua y densidad de larvas, pero dado que estas variables no fueron medidas experimentalmente, fue imposible cuantificar el efecto de esta fuente de variación (Falconer y Mackay 1996). Estas mismas razones, podrían hacer que el efecto de la familia se reduzca y, por lo tanto, se subestime la $\mathrm{H}^{2}$ en sentido amplio.

Los machos y las hembras de una misma especie a menudo difieren en los rasgos relacionados con la morfología, la fisiología y el comportamiento, y se cree que tales disparidades surgen de la sincronización de la asignación de recursos, especialmente con respecto a la reproducción (Badyaev 2002). Frecuentemente, los machos buscarán y competirán por las hembras, mientras que las hembras elegirán entre los machos competidores y generarán la descendencia (Petrie et al. 1991; Miller y Svensson 2014). Tales antecedentes generales conducen a inversiones desproporcionadas en rasgos entre los sexos, con las hembras invirtiendo relativamente más en su aparato productivo y en la cría en la mayoría de los casos (Thornhill y Alcock 1983; Olof et al. 1994; Miller y Svensson 2014). Esto podría explicar el dimorfismo sexual encontrado para los promedios de la longitud, el alargamiento, los tamaños de las manchas costales y en la tasa promedio $\mathrm{PB} / \mathrm{ta}^{-} \mathrm{III}_{2}$ (Anexo 2). En el 29,2\% de los casos con dimorfismo sexual se favorece la mayor longitud de las manchas oscuras en hembras mientras que en el restante porcentaje se favorece la mayor longitud de las manchas claras en machos.
El efecto de la temperatura para la tasa SCP/DSD podría explicarse bajo el supuesto de considerar que el fenotipo más estable se da a $24{ }^{\circ} \mathrm{C}$, en donde el tamaño de estas dos manchas es antagónico (mayor longitud de DSD menor longitud $\mathrm{SCP}$ o viceversa). Esta relación es muy sensible a la temperatura como lo propusieron Sueur et al. (1992). A 20 o $28^{\circ} \mathrm{C}$ se estabiliza mostrando en algunas familias relaciones fenotípicas directas significativas y en otras no.

Los aportes más altos del factor familia para PHD, HD, y las proporciones HP/PHD, SCP/DSD (42,5\%) y PB/ta-III podrían indicar que el aporte genético sobre la variación fenotípica es significativo, independientemente de la temperatura donde se criaron las progenies. Por otro lado, el hecho de que se presente significancia en este componente entre tasas también podría indicar covariación genética significativa en estas características, lo cual podría explicarse por acción pleiotrópica o epítasis de genes involucrados en la pareja de caracteres. Esto último, también podría explicar porque la temperatura muestra un mayor efecto sobre la fusión SP + PRSD + ASP $(69,4 \%)$ que, sobre cada una de las manchas individuales, sobre las cuales el efecto no fue significativo (SP y ASP) y sólo aportó el 2,8 \% a la variación fenotípica de PRSD.

\section{Conclusiones}

Independientemente de la temperatura de crianza de las larvas, las progenies de familias de Nys. triannulatus del sur de Colombia obtenidas en cámaras ambientales reguladas entre 20 y $28^{\circ} \mathrm{C}$, muestran alta frecuencia de solamente uno de los patrones costales (I) y dimorfismo sexual para la tasa largo/ ancho de las alas, la cual es significativamente mayor en machos.

En cada una de las temperaturas de crianza se presenta dimorfismo sexual en muchos de los otros caracteres analizados. Dependiendo del sexo, temperaturas más altas favorecen el aumento de la longitud de las manchas claras u oscuras. En hembras, en casi la tercera parte de los casos, se favorece la mayor longitud de las manchas oscuras, mientras que, en machos, aproximadamente las dos terceras partes de los casos, presentan mayor longitud de las manchas claras.

En general, en las familias, la temperatura influye sobre los tamaños de las manchas costales y tasas que relacionan algunas manchas vecinas, pero el efecto de los sexos sobre la variación fenotípica de los caracteres es significativo solamente para algunos de estos, presentándose pocas interacciones significativas entre temperatura, familia y sexo. Sin embargo, es importante resaltar, que la longitud de las alas también varía significativamente entre progenies de una misma temperatura y entre familias de individuos. Por otro lado, el aporte genético al tamaño, así como de las familias, a la variación fenotípica, es significativo en casi todos los caracteres que fueron analizados en las alas de $N$. triannulatus.

\section{Agradecimientos}

Agradecemos a la Universidad del Valle y a su Vicerrectoría de Investigaciones por la financiación concedida a los autores a través del proyecto No. 71106, y a la Vicedecanatura de Investigaciones de la Facultad de Ciencias Naturales y Exactas por su apoyo en los trámites de ejecución. A Jefferson Panche por su invaluable ayuda en el trabajo de recolección de ejemplares, así como a las personas de Puerto Asís quienes colaboraron en el proceso de obtención de las muestras de 
Anopheles. A Nelson Toro y la sección de Genética del Depto. de Biología de la Universidad del Valle. HCH y RGO, agradecen al Departamento de Biología, Facultad de Ciencias Naturales y Exactas, y Vicerrectoría de Investigaciones, Universidad del Valle, Santiago de Cali, Colombia, por su continuo soporte y apoyo.

\section{Literatura citada}

ARAÚJO, M. da-S.; GIL, L. H. S.; E-SILVA, A. de-A. 2012. Larval food quantity affects development time, survival and adult biological traits that influence the vectorial capacity of Anopheles darlingi under laboratory conditions. Malaria Journal 11: 261. https://doi.org/10.1186/1475-2875-11-261

BADYAEV, A. V. 2002. Growing apart: An ontogenetic perspective on the evolution of sexual size dimorphism. Trends in Ecology \& Evolution 17 (8): 369-378. https://doi.org/10.1016/S01695347(02)02569-7

BELEN, A.; ALTEN, B.; AYTEKIN, A. M. 2004. Altitudinal variation in morphometric and molecular characteristics of Phlebotomus papatasi populations. Medical and Veterinary Entomology 18 (4): 343-350. https://doi.org/10.1111/j.0269283X.2004.00514.x

BLAND J. M.; ALTMAN, D. G. 1995. Multiple significance tests: the Bonferroni method. British Medical Journal 310-170. https:// doi.org/10.1136/bmj.310.6973.170

BOMPHREY, R.; NAKATA, T.; PHILLIPS, N.; WALKER, S. M. 2017. Smart wing rotation and trailing-edge vortices enable high frequency mosquito flight. Nature 544: 92-95. https://doi. org/10.1038/nature21727

BOURKE, P. M.; VOORRIPS, R. E.; VISSERET, R. G. F.; MALIEPAARD, C. 2018. Tools for genetic studies in experimental populations of polyploids. Frontiers in Plant Science 9: 1-19. https://doi.org/10.3389/fpls.2018.00513

BUSTAMANTE, D. M.; MONROY, C.; MENES, M.; RODAS, A.; SALAZAR-SCHETTINO, P. M.; ROJAS, G.; PINTO, N.; GUHL, F.; DUJARDIN, J. P. 2004. Metric variation among geographic populations of the Chagas vector Triatoma dimidiata (Hemiptera: Reduviidae: Triatominae) and related species. Journal of Medical Entomology 41 (3): 296-301. https://doi. org/10.1603/0022-2585-41.3.296

CALLE L., D. A.; QUIÑONES, M. L.; ERAZO, H. F.; JARAMILLO O., N. 2002. Morphometric discrimination of females of five species of Anopheles of the subgenus Nyssorhynchus from Southern and Northwest Colombia. Memorias do Instituto Oswaldo Cruz 97 (8): 1191-1195. https://doi.org/10.1590/S007402762002000800021

CAZORLA P., D. 2009. Multivariate morphometric differentiation between females of two cryptic species of Lutzomyia subgenus Helcocyrtomyia (Diptera: Psychodidae). Revista Colombiana de Entomología 35 (2): 197-201. ISSN 0120-0488. http://www. scielo.org.co/pdf/rcen/v35n2/v35n2a15.pdf

CERÓN-MUÑOZ, H. D.; COSIN, R.; COIMBRA, R. F. F.; CORREA, L. G. N.; CATALANO, F. M. 2013. Experimental investigation of wing-tip devices on the reduction of induced drag. Journal of Aircraft 50 (2): 441-449. https://doi.org/10.2514/1. C031862

CHARLWOOD, J. D. 1996. Biological variation in Anopheles darlingi Root. Memorias do Instituto Oswaldo Cruz 91 (4): 391-398.

DANTUR JURI, M. J.; LIRIA, J.; NAVARRO, J. C.; RODRÍGUEZ, R.; FRITZ, G. N. 2011. Morphometric variability of Anopheles pseudopunctipennis (Diptera: Culicidae) from different ecoregions of Argentina and Bolivia. Florida Entomologist 94 (3): 428-438. https://doi.org/10.1653/024.094.0307

DOS SANTOS SILVA, J.; CARBAJAL DE LA FUENTE, A. L.; SERRA-FREIRE, N. M.; GUIMARAES, A. E.; SOARES SARMENTO, J.; ALENCAR, J. 2012. Morphometric analysis of Chagasia fajardi (Diptera: Culicidae) populations in Brazil.
Revista Colombiana de Entomología 38 (2): 276-281. http:// www.scielo.org.co/pdf/rcen/v38n2/v38n2a19.pdf

DUDLEY, R. 2002. The biomechanics of insect flight: Form, function, evolution. Princeton, New Jersey: Princeton University Press, EE. UU. 476 p. https://doi.org/10.2307/j.ctv301g2x

DUJARDIN, J. P. 2011. 16 - Modern morphometrics of medically important insects. pp. 473-501. En: Tibayrenc, M. (Ed.). Genetics and evolution of infectious disease. Elsevier. Londres, Inglaterra. 772 p. https://doi.org/10.1016/B978-0-12-384890$1.00016-9$

FAJARDO RAMOS, M.; GONZÁLEZ OBANDO, R.; SUÁREZ, M. F.; LÓPEZ, D.; WILKERSON, R.; SALLUM, M. A. M. 2008. Morphological analysis of three populations of Anopheles (Nyssorhynchus) nuneztovari Gabaldón (Diptera: Culicidae) from Colombia. Memorias do Instituto Oswaldo Cruz 103 (1): 85-92. https://doi.org/10.1590/S0074-02762008000100013

FALCONER, D. S.; MACKAY, T. F. C. 1996. Introduction to quantitative genetics. Longman Scientific and Technical. Essex, Reino Unido. $464 \mathrm{p}$.

FARAN, M. E. 1980. Mosquito studies (Diptera: Culicidae) XXXIV. A revision of the Albimanus section of the subgenus Nyssorhynchus of Anopheles. American Entomology Institute 15 (7): 1-216. http://mosquito-taxonomic-inventory.info/sites/ mosquito-taxonomic-inventory.info/files/Faran\%201980.pdf

FARAN, M. E.; LINTHICUM, K. J. 1981. A handbook of the Amazonian species of Anopheles (Nyssorhynchus) (Diptera, Culicidae). Mosquito Systematic 13 (1): 1-81. http://mosquito-taxonomic-inventory.info/sites/mosquito-taxonomic-inventory.info/ files/Faran\%20\%26\%20Linthicum\%201981.pdf

FOSTER, P. G.; OLIVEIRA, T. M.; BERGO, E. S.; CONN, J. E.; SANTANA, D. C.; NAGAKI, S. S.; NIHEI, S.; LAMAS, C. E.; GONZÁlEZ, C.; MOREIRA, C. C.; MUREB SALLUM, M. A. 2017. Phylogeny of Anophelinae using mitochondrial protein coding genes. The Royal Society Open Science 4: 170758. https://doi.org/10.1098/rsos.170758

FREEMAN, M. F.; TUKEY, J. W. 1950. Transformations related to the angular and the square root. The Annals of Mathematical Statistics 21 (4): 607-611. https://doi.org/10.1214/ aoms/1177729756

FUSCO, G.; MINELLI, A. 2010. Phenotypic plasticity in development and evolution: facts and concepts. Philosophical Transactions of the Royal Society Biological 365: 547-556. https://doi.org/10.1098/rstb.2009.0267

GÓMEZ, G. F.; CORREA, M. M. 2017. Discrimination of Neotropical Anopheles species based on molecular and wing geometric morphometric traits. Infection, Genetics and Evolution 54: 379386. https://doi.org/10.1016/j.meegid.2017.07.028

GÓMEZ, G.; JARAMILLO, L.; CORREA, M. M. 2013. Wing geometric morphometrics and molecular assessment of members in the Albitarsis complex from Colombia. Molecular Ecology Resources 13 (6): 1082-1092. https://doi.org/10.1111/17550998.12126

GÓMEZ, G. F.; MÁRQUEZ, E. J.; GUTIÉRREZ, L. A.; CONN, J. E.; CORREA, M. M. 2014. Geometric morphometric analysis of Colombian Anopheles albimanus (Diptera: Culicidae) reveals significant effect of environmental factors on wing traits and presence of a metapopulation. Acta Tropica 135: 75-85. https:// doi.org/10.1016/j.actatropica.2014.03.020

GÓMEZ, G. F.; BICKERSMITH, S. A.; GONZÁLEZ, R.; CONN, J. E.; CORREA, M. M. 2015. Molecular taxonomy provides new insights into Anopheles species of the neotropical Arribalzagia series. PLoS ONE 10 (3): e0119488. https://doi.org/10.1371/ journal.pone.0119488

GONZÁLEZ, R.; CARREJO, N.; WILKERSON, R. C.; ALARCON, J.; ALARCON-ORMASA, J.; RUIZ, F.; BHATIA, R.; LOAIZA, J.; LINTON, Y-M. 2010. Confirmation of Anopheles (Anopheles) calderoni Wilkerson, 1991 (Diptera: Culicidae) in Colombia and Ecuador through molecular and morphological correlation with topotypic material. Memorias do Instituto Os- 
waldo Cruz 105 (8): 1001-1019. https://doi.org/10.1590/S007402762010000800009

GONZÁLEZ OBANDO, R.; CARREJO GIRONZA, N. S. 2009. Introducción al estudio taxonómico de Anopheles de Colombia: claves y notas de distribución. 2nd ed., Universidad del Valle; Cali, Colombia. 260 p. https://doi.org/10.2307/j.ctv14jx88t

HRIBAR, L. J. 1995. Costal wing spot variations within and among progeny of single female Anopheles nuneztovari (Diptera: Culicidae). Mosquito Systematics 27: 1-10.

KIHLBERG, J. K.; HERSON, J. H.; SCHOTZ, W. E. 1972. Square root transformation revisited. Journal of Statistics Society 21 (1): 76-81. https://doi.org/10.2307/2346609

LANCIANI, C. A.; LE, T. M. 1995. Effect of temperature on the wing length body weight relationship in Anopheles quadrimaculatus. Journal of the American Mosquito Control Association 11 (2): 241-243.

LEVENE, H. 1960. Robust tests for equality of variances. pp. 278292. En: Olkin, I.; Ghurye, S. G.; Hoeffding, W.; Madow, W. G.; Mann, H. B. (Eds.). Contributions to probability and statistics: essays in honor of harold hotelling. Stanford University Press. California, EE. UU. 517 p.

LINTHICUM, K. J. 1988. A revision of the Argyritarsis section of the subgenus Nyssorhynchus of Anopheles (Diptera: Culicidae). Mosquito Systematic 20 (2): 98-174. http://mosquito-taxonomic-inventory.info/sites/mosquito-taxonomic-inventory.info/files/ Linthicum\%201988.pdf

LORENZ, C.; MARQUES, T. C.; SALLUM, M. A. M.; SUESDEK, L. 2014. Altitudinal population structure and microevolution of the malaria vector Anopheles cruzii (Diptera: Culicidae). Parasites and Vectors 7: 581. https://doi.org/10.1186/s13071-014-0581-8

MARÍN LONDOÑO, O. A. 2014. Variación morfométrica en el patrón alar de Anopheles triannulatus de cuatro poblaciones de Colombia. Boletín del Museo de Entomología de la Universidad del Valle 15 (2): 17-32.

MILLER, C. W.; SVENSSON, E. I. 2014. Sexual selection in complex environments. The Annual Review of Entomology 59: 427445. https://doi.org/10.1146/annurev-ento-011613-162044

MORENO, M.; BICKERSMITH, S.; HARLOW, W.; HILDEBRANDT, J.; McKEON, S. N.; SILVA-DO-NASCIMENTO, T. F.; LOAIZA, J. R.; RUÍZ, F.; LOURENÇO-DE-OLIVEIRA, R.; SALLUM, M. A. M.; BERGO, E. S.; FRITZ, G. N.; WILKERSON, R. C.; LINTON, Y. M.; DANTUR JURI, M. J.; RANGEL, Y.; PÓVOA, M. M.; GUTIÉRREZ-BUILES, L. A.; CORREA, M. M.; CONN, J. E. 2013. Phylogeography of the neotropical Anopheles triannulatus complex (Diptera: Culicidae) supports deep structure and complex patterns. Parasites and Vectors 6: 47-64. https://doi.org/10.1186/1756-3305-6-47

MOTOKI, M. T.; SUESDEK, L.; STERLINO BERGO, E.; MUREB SALLUM, M. A. 2012. Wing geometry of Anopheles darlingi Root (Diptera: Culicidae) in five major Brazilian ecoregions. Infection, Genetics and Evolution 12 (6): 1246-1252. https://doi. org/10.1016/j.meegid.2012.04.002

MÜLLER, G. B. 2007. Evo-devo: extending the evolutionary synthesis. Nature Reviews Genetics 8 (12): 943-949. https://doi. org/10.1038/nrg2219

NORBERG, U. M. 1995. How a long tail and changes in mass and wing shape affect the cost for flight in animals. Functional Ecology 9 (1): 48-54. https://doi.org/10.2307/2390089

OLOF, L.; BENGT, K.; CHRISTER, W. 1994. Unpredictable food and sexual size dimorphism in insects. Proceedings of the Royal Society of London, Series B 258: 121-125. https://doi. org/10.1098/rspb.1994.0151

PACHECO, M. A.; GONZÁLEZ, R.; BROCHERO, H. L. 2017. Anopheles darlingi (Diptera: Culicidae) Root 1926: variaciones morfométricas en alas y patas de poblaciones de Colombia. Biomédica 37 (Suppl. 2): 124-134. https://doi.org/10.7705/biomedica.v37i0.3492

PACHECO-GÓMEZ, M. A.; GONZÁLEZ-OBANDO, R.; BROCHERO, H. L. 2018. Morphometric variations of two populations of Anopheles albitarsis F (Diptera: Culicidae) in the Orinoquia region, Colombia. Revista de Facultad de Medicina 66 (2): 201-208. http://doi.org/10.15446/revfacmed.v66n2.61071

PETRARCA, V.; SABATELLI, G.; TOURÉ, Y. T; DI DIDECO, M. A. 1998. Morphometric multivariate analysis in Anopheles gambiae s.s. (Diptera: Culicidae), Journal of Medical Entomology 35 (1): 16-25. https://doi.org/10.1093/jmedent/35.1.16

PETRIE, M.; HALLIDAY, T.; SANDERS, C. 1991. Peahens prefer peacocks with elaborate trains. Animal Behaviour 41 (2): 323331. https://doi.org/10.1016/S0003-3472(05)80484-1

RICHARDSON, J. B.; JAMESON, S. B.; GLORIA-SORIA, A.; WESSON, D. M.; POWELL, J. 2015. Evidence of limited polyandry in a natural population of Aedes aegypti. The American Journal of Tropical Medicine and Hygiene 93 (1): 189-193. http://doi.org/10.4269/ajtmh.14-0718

RODRÍGUEZ-ZABALA, J.; GONZÁLEZ, R.; CORREA, M. M.; GÓMEZ, G. F. 2016. Análisis morfométrico de dos poblaciones de Anopheles (Anopheles) calderoni (Diptera: Culicidae) del suroccidente colombiano. Revista Mexicana de Biodiversidad 87 (3): 966-971. http://doi.org/10.1016/j.rmb.2016.06.005

ROSA-FREITAS, M. G.; DEANE, L. M.; MOMEN, H. 1990. A morphological, isoenzymatic and behavioural study of ten populations of Anopheles (Nyssorhynchus) albitarsis LynchArribalzaga, 1878 (Diptera: Culicidae) including from the type locality - Baradero, Argentina. Memorias do Instituto Oswaldo Cruz 85 (3): 275-289. https://doi.org/10.1590/S007402761990000300004

RUBIO-PALIS, Y. 2000. Anopheles (Nyssorhynchus) de Venezuela Taxonomía, bionomía, ecología e importancia médica. Escuela de Malariología y Saneamiento Ambiental "Dr. Arnoldo Gabaldón". Maracay, Venezuela. 120 p.

SARAIVA, J. F.; SOUTO, R. N. P.; SCARPASSA, V. M. 2018. Molecular taxonomy and evolutionary relationships in the Oswaldoi-Konderi complex (Anophelinae: Anopheles: Nyssorhynchus) from the Brazilian Amazon region. PLoS ONE 13 (3): e0193591. https://doi.org/10.1371/journal.pone.0193591

SCARPASSA, V. M.; CUNHA-MACHADO, A. S.; SARAIVA, J. F. 2016. Evidence of new species for malaria vector Anopheles nuneztovari sensu lato in the Brazilian Amazon region. Malaria Journal 15: 205. https://doi.org/10.1186/s12936-016-1217-6

SHUTT, B.; STABLES, L.; ABOAGYE-ANTWI, F.; MORAN, J.; TRIPET, F. 2010. Male accessory gland proteins induce female monogamy in anopheline mosquitoes. Medical and Veterinary Entomology 24 (1): 91-94. https://doi.org/10.1111/j.13652915.2009.00849.x

SILVA DO NASCIMENTO, T. F.; LOURENCO-DE-OLIVEIRA, R. 2002. Anopheles halophylus, a new species of the subgenus Nyssorhynchus (Diptera: Culicidae) from Brazil. Memorias do Instituto Oswaldo Cruz 97 (6): 801-811. https://doi.org/10.1590/ S0074-02762002000600010

SILVA-DO-NASCIMENTO, T. F.; WILKERSON, R. C.; LOURENÇO-DE-OLIVEIRA, R.; MONTEIRO, F. A. 2006. Molecular confirmation of the specific status of Anopheles halophylus (Diptera: Culicidae) and evidence of a new cryptic species within An. triannulatus in Central Brazil. Journal of Medical Entomology 43 (3): 455-459. https://doi.org/10.1093/ jmedent $/ 43.3 .455$

SUEUR, D. L.; SHARP, B. L.; APPLETON, C. C. 1992. Darkscaled areas on adult Anopheles mosquitoes are selectively affected by temperature-related size variation. Medical and Veterinary Entomology 6 (4): 396-398. https://doi. org/10.1111/j.1365-2915.1992.tb00641.x

THORNHILL, R.; ALCOCK, J. 1983. The evolution of insect mating systems. Harvard University Press, Cambridge, EE. UU. 547 p.

TRIPET, F.; TOURE, Y. T.; DOLO, G.; LANZARO, G. C. 2003. Frequency of multiple inseminations in field-collected Anopheles gambiae females revealed by DNA analysis of transferred sperm. The American Journal Tropical Medicine and Hygiene 68 (1): 1-5. https://doi.org/10.4269/ajtmh.2003.68.1.0680001 
WILKERSON, R. C.; PEYTON, E. L. 1990. Standardized nomenclature for the costal wing spots of the genus Anopheles and other spotted-wing mosquitoes (Diptera: Culicidae). Journal of Medical Entomology 27 (2): 207-224. https://doi.org/10.1093/ jmedent/27.2.207

\section{$\overline{\text { Origen y financiación }}$}

Este manuscrito fue originado en el proyecto de investigación (No. 71106), titulado "Efecto de la temperatura en la expresión fenotipica del área costal de Anopheles (Nyssorhynchus) triannulatus (Diptera: Culicidae: Anophelinae), usando cámaras ambientadas diseñadas en la Universidad del Valle”. Este fue financiado por la Universidad del Valle, presupuesto asignado mediante convocatoria interna.

\section{Contribución de los autores}

Ranulfo González Obando: Verificación taxonómica, fotografias, realización de mediciones, colaboración en la interpretación de los análisis y escritura del manuscrito. Coordinación de las actividades de revisión del manuscrito.

Heiber Cárdenas Henao: Realización de mediciones, análisis, interpretación y escritura del manuscrito.

Omar Alejandro Marín-Londoño. Recolección, cría y manejo del material biológico, preparación de placas, fotografías, ayuda en el análisis de los datos y escritura del manuscrito. Todos los autores aprobaron la versión final del manuscrito.

Anexo 1. Números de hembras $\left(\mathrm{n}_{1}\right)$ y machos $\left(\mathrm{n}_{2}\right)$, promedios y desviaciones estándar de la longitud (mm), de los tamaños de manchas costales $(*)$, de las proporciones HP/PHD, SCP/DSD y (PB/ta-III $)$ y de la fusión SP + PRSD + ASP, en las alas derechas de las progenies hembras y machos de Nys. triannulatus, criadas a tres temperaturas.

\begin{tabular}{|c|c|c|c|c|c|c|c|c|c|c|c|c|c|c|c|c|c|c|}
\hline \multirow{3}{*}{$\begin{array}{c}\text { Temperatura } \\
\text { Característica }\end{array}$} & \multicolumn{6}{|c|}{$20^{\circ} \mathrm{C}$} & \multicolumn{6}{|c|}{$24^{\circ} \mathrm{C}$} & \multicolumn{6}{|c|}{$28^{\circ} \mathrm{C}$} \\
\hline & \multicolumn{3}{|c|}{ Hembras (H) } & \multicolumn{3}{|c|}{ Machos (M) } & \multicolumn{3}{|c|}{ Hembras (H) } & \multicolumn{3}{|c|}{ Machos (M) } & \multicolumn{3}{|c|}{ Hembras (H) } & \multicolumn{3}{|c|}{ Machos (M) } \\
\hline & $n_{1}$ & $\bar{X}$ & D.E. & $\mathbf{n}_{2}$ & $\bar{X}$ & D.E. & $\mathrm{n}_{1}$ & $\bar{X}$ & D.E. & $\mathbf{n}_{2}$ & $\bar{X}$ & D.E. & $n_{1}$ & $\bar{X}$ & D.E. & $n_{2}$ & $\bar{X}$ & D.E. \\
\hline Longitud (mm) & 23 & 2,829 & 0,383 & 10 & 2,746 & 0,393 & 30 & 2,749 & 0,305 & 21 & 2,73 & 0,277 & 22 & 2,683 & 0,286 & 6 & 2,529 & 0,224 \\
\hline $\mathrm{BP}+\mathrm{PHP}^{*}$ & 15 & 0,458 & 0,026 & 6 & 0,45 & 0,048 & 18 & 0,461 & 0,034 & 14 & 0,453 & 0,052 & 19 & 0,462 & 0,03 & 6 & 0,474 & 0,028 \\
\hline PHD* & 21 & 0,427 & 0,063 & 8 & 0,445 & 0,053 & 28 & 0,418 & 0,043 & 18 & 0,429 & 0,045 & 22 & 0,457 & 0,043 & 6 & 0,481 & 0,032 \\
\hline HP* & 21 & 0,474 & 0,047 & 10 & 0,487 & 0,023 & 29 & 0,492 & 0,027 & 19 & 0,492 & 0,026 & 22 & 0,475 & 0,02 & 6 & 0,482 & 0,03 \\
\hline $\mathrm{HD}^{*}$ & 22 & 0,444 & 0,042 & 9 & 0,45 & 0,048 & 30 & 0,441 & 0,038 & 20 & 0,455 & 0,032 & 22 & 0,466 & 0,031 & 6 & 0,473 & 0,023 \\
\hline PSP* & 22 & 0,436 & 0,031 & 9 & 0,434 & 0,02 & 30 & 0,443 & 0,042 & 20 & 0,426 & 0,029 & 22 & 0,438 & 0,025 & 6 & 0,46 & 0,013 \\
\hline PSD* & 22 & 0,549 & 0,047 & 10 & 0,573 & 0,038 & 29 & 0,554 & 0,046 & 20 & 0,584 & 0,037 & 22 & 0,55 & 0,032 & 6 & 0,552 & 0,07 \\
\hline SP* & 22 & 0,44 & 0,046 & 10 & 0,436 & 0,037 & 29 & 0,436 & 0,022 & 21 & 0,437 & 0,031 & 22 & 0,427 & 0,029 & 6 & 0,466 & 0,046 \\
\hline PRSD* & 21 & 0,427 & 0,025 & 9 & 0,426 & 0,03 & 29 & 0,429 & 0,036 & 21 & 0,426 & 0,025 & 22 & 0,445 & 0,023 & 6 & 0,444 & 0,037 \\
\hline ASP* & 21 & 0,425 & 0,034 & 9 & 0,448 & 0,032 & 29 & 0,454 & 0,064 & 21 & 0,453 & 0,04 & 22 & 0,443 & 0,024 & 6 & 0,456 & 0,024 \\
\hline $\mathrm{DSD}^{*}$ & 22 & 0,679 & 0,046 & 10 & 0,676 & 0,033 & 29 & 0,69 & 0,057 & 21 & 0,651 & 0,041 & 22 & 0,713 & 0,042 & 6 & 0,713 & 0,035 \\
\hline $\mathrm{SCP}^{*}$ & 23 & 0,461 & 0,022 & 10 & 0,474 & 0,021 & 29 & 0,453 & 0,037 & 21 & 0,482 & 0,049 & 22 & 0,449 & 0,019 & 6 & 0,465 & 0,019 \\
\hline $\mathrm{PD}^{*}$ & 23 & 0,684 & 0,058 & 10 & 0,688 & 0,025 & 27 & 0,686 & 0,064 & 19 & 0,648 & 0,038 & 22 & 0,702 & 0,026 & 6 & 0,663 & 0,096 \\
\hline PP* & 20 & 0,489 & 0,026 & 9 & 0,496 & 0,023 & 24 & 0,507 & 0,028 & 18 & 0,512 & 0,033 & 19 & 0,486 & 0,019 & 5 & 0,526 & 0,043 \\
\hline $\mathrm{AD}^{*}$ & 19 & 0,432 & 0,037 & 9 & 0,44 & 0,042 & 19 & 0,441 & 0,028 & 16 & 0,423 & 0,033 & 17 & 0,46 & 0,036 & 2 & 0,46 & 0,018 \\
\hline $\mathrm{AP} *$ & 18 & 0,454 & 0,029 & 8 & 0,471 & 0,028 & 22 & 0,458 & 0,028 & 20 & 0,444 & 0,039 & 17 & 0,45 & 0,027 & 2 & 0,483 & 0,01 \\
\hline $\mathrm{HP} / \mathrm{PHD}^{* *}$ & 18 & 1,534 & 0,392 & 8 & 1,458 & 0,328 & 24 & 1,622 & 0,335 & 13 & 1,616 & 0,326 & 21 & 1,285 & 0,285 & 6 & 1,181 & 0,089 \\
\hline $\mathrm{SCP} / \mathrm{DSD}^{* *}$ & 21 & 0,78 & 0,056 & 10 & 0,798 & 0,048 & 29 & 0,762 & 0,076 & 21 & 0,841 & 0,118 & 22 & 0,732 & 0,037 & 6 & 0,743 & 0,017 \\
\hline$(\mathrm{SP}+\mathrm{PRSD}+\mathrm{ASP})^{*}$ & 21 & 0,808 & 0,062 & 10 & 0,794 & 0,023 & 29 & 0,928 & 0,05 & 21 & 0,945 & 0,058 & 22 & 0,801 & 0,026 & 6 & 0,814 & 0,054 \\
\hline $\mathrm{PB} / \mathrm{ta}^{-\mathrm{III}_{2}} * *$ & 22 & 0,815 & 0,058 & 8 & 0,819 & 0,029 & 24 & 0,833 & 0,051 & 17 & 0,829 & 0,049 & 14 & 0,893 & 0,059 & 5 & 0,926 & 0,034 \\
\hline
\end{tabular}

D.E.: Desviación Estándar; (*) Datos transformados según Freeman y Tukey (1950) y ** según Kihlberg et al. (1972) 
Anexo 2. Pruebas t-student con su significancia (p) para comparar longitud promedio y tamaños promedio de las manchas costales-ala derecha $(*)$ y tasa promedio de la mancha oscura basal/longitud del tarsómero posterior dos $\left(\mathrm{PB} / \mathrm{ta}-\mathrm{III}_{2}\right)$ de progenies hembras $(\mathrm{H})$ y machos $(\mathrm{M})$ en cada familia de Nys. triannulatus. Temperaturas: (a) $20^{\circ} \mathrm{C}$; (b) $24^{\circ} \mathrm{C}$; (c) $28^{\circ} \mathrm{C}$.

(a) Temperatura $20^{\circ} \mathrm{C}$.

\begin{tabular}{|c|c|c|c|c|c|c|c|c|c|c|c|c|c|c|c|c|}
\hline \multirow{2}{*}{ Caracter } & \multicolumn{4}{|c|}{ Familia 1} & \multicolumn{4}{|c|}{ Familia 2} & \multicolumn{4}{|c|}{ Familia 3} & \multicolumn{4}{|c|}{ Familia 4} \\
\hline & $\begin{array}{c}\mathrm{n} \\
(\mathrm{H}+\mathrm{M})\end{array}$ & H-M & $\mathbf{t}$ & $\mathbf{p}$ & $\begin{array}{c}n \\
(H+M)\end{array}$ & H-M & $\mathbf{t}$ & $\mathbf{p}$ & $\begin{array}{c}\text { n } \\
\mathbf{H}+\mathbf{M})\end{array}$ & H-M & $t$ & $\mathbf{p}$ & $\begin{array}{c}n \\
H+M) \\
\end{array}$ & H-M & $\mathbf{t}$ & $\mathbf{p}$ \\
\hline $\begin{array}{l}\text { Longitud } \\
(\mathrm{mm})\end{array}$ & 7 & 0,570 & 13,80 & $<0,0001$ & 7 & 0,611 & 35,24 & $<0,0001$ & 10 & 0,1081 & 9,14 & $<0,0001$ & 9 & 0,030 & 2,43 & 0,0453 \\
\hline $\mathrm{BP}+\mathrm{PHP}^{*}$ & 4 & 0,109 & 14,36 & 0,0048 & 6 & 0,871 & 562,45 & $<0,0001$ & 6 & $-0,033$ & $-6,72$ & 0,0026 & 5 & 0,007 & 2,17 & 0,1180 \\
\hline PHD* & 7 & $-0,032$ & $-6,77$ & 0,0011 & 6 & 0,880 & 344,70 & $<0,0001$ & 8 & $-0,003$ & $-1,19$ & 0,2782 & 8 & $-0,042$ & $-8,35$ & 0,0002 \\
\hline $\mathrm{HP}^{*}$ & 7 & 0,005 & 1,01 & 0,3599 & 7 & 0,887 & 270,91 & $<0,0001$ & 8 & $-0,002$ & $-2,23$ & 0,0672 & 9 & $-0,041$ & $-6,97$ & 0,0002 \\
\hline $\mathrm{HD}^{*}$ & 6 & $-0,020$ & $-3,78$ & 0,0194 & 7 & $-0,029$ & $-10,55$ & $<0,0001$ & 9 & $-0,005$ & $-2,86$ & 0,0243 & 9 & $-0,006$ & $-3,72$ & 0,0074 \\
\hline PSP* & 6 & $-0,002$ & $-0,28$ & 0,7963 & 7 & 0,008 & 2,64 & 0,0463 & 9 & $-0,005$ & $-3,33$ & 0,0127 & 9 & $-0,004$ & $-2,94$ & 0,0218 \\
\hline PSD* & 7 & $-0,044$ & $-7,75$ & 0,0006 & 7 & $-0,023$ & $-8,83$ & 0,0003 & 9 & $-0,013$ & $-8,70$ & 0,0001 & 9 & $-0,025$ & $-5,31$ & 0,0011 \\
\hline SP* & 7 & $-0,002$ & $-0,44$ & 0,6794 & 7 & 0,013 & 4,22 & 0,0083 & 9 & $-0,026$ & $-8,11$ & 0,0001 & 9 & 0,027 & 4,66 & 0,0023 \\
\hline PRSD* & 7 & $-0,018$ & $-4,00$ & 0,0103 & 6 & $-0,011$ & $-6,78$ & 0,0025 & 8 & 0,0053 & 3,67 & 0,0104 & 9 & 0,025 & 16,30 & $<0,0001$ \\
\hline ASP* & 7 & $-0,056$ & $-16,85$ & $<0,0001$ & 6 & 0,005 & 1,24 & 0,2845 & 8 & 0,0013 & 0,82 & 0,4413 & 9 & $-0,027$ & $-27,17$ & $<0,0001$ \\
\hline DSD* & 7 & 0,002 & 0,20 & 0,8483 & 7 & 0,024 & 3,73 & 0,0136 & 9 & $-0,014$ & $-5,20$ & 0,0013 & 9 & $-0,001$ & $-1,43$ & 0,1956 \\
\hline $\mathrm{SCP}^{*}$ & 7 & $-0,019$ & $-4,28$ & 0,0078 & 7 & $-0,016$ & $-8,79$ & 0,0003 & 10 & $-0,016$ & $-7,61$ & 0,0001 & 9 & $-0,003$ & $-1,26$ & 0,2482 \\
\hline $\mathrm{PD}^{*}$ & 7 & 0,014 & 1,98 & 0,1052 & 7 & $-0,004$ & $-0,96$ & 0,3808 & 10 & 0,0029 & 1,99 & 0,0816 & 9 & $-0,027$ & $-4,36$ & 0,0033 \\
\hline PP* & 7 & $-0,021$ & $-5,37$ & 0,0030 & 7 & 0,004 & 0,96 & 0,3804 & 9 & $-0,012$ & $-10,54$ & 0,0000 & 6 & 0,004 & 1,20 & 0,2968 \\
\hline $\mathrm{AD}^{*}$ & 6 & $-0,019$ & $-3,41$ & 0,0270 & 7 & $-0,007$ & $-2,02$ & 0,0996 & 9 & $-0,010$ & $-5,19$ & 0,0013 & 6 & 0,008 & 4,30 & 0,0126 \\
\hline $\mathrm{AP}^{*}$ & 5 & 0,003 & 0,91 & 0,4307 & 7 & $-0,025$ & $-15,22$ & $<0,0001$ & 8 & $-0,016$ & $-8,40$ & 0,0002 & 6 & $-0,012$ & $-5,14$ & 0,0068 \\
\hline HP/PHD** & 6 & 0,153 & 6,39 & 0,0031 & 6 & 0,166 & 6,60 & 0,0027 & 8 & 0,0797 & 1,83 & 0,1172 & 6 & 0,214 & 7,07 & 0,0021 \\
\hline SCP/DSD** & 6 & $-0,020$ & $-3,05$ & 0,0379 & 7 & $-0,039$ & $-7,29$ & 0,0008 & 9 & $-0,010$ & $-1,82$ & 0,1124 & 9 & $-0,003$ & $-0,56$ & 0,5922 \\
\hline $\begin{array}{l}(\mathrm{SP}+\mathrm{PRSD}+ \\
\mathrm{ASP})^{*}\end{array}$ & 6 & $-0,029$ & - 11,97 & 0003 & 7 & 0,000 & 0,00 & 3 & 9 & 0,0354 & 15,85 & $<0,0001$ & 9 & 0,037 & 5,25 & 0,0012 \\
\hline $\mathrm{PB} / \mathrm{ta}-\mathrm{III}_{2} * *$ & 4 & - & - & - & 7 & 0,015 & 8,67 & 0,0003 & 10 & $-0,028$ & $-4,40$ & 0,0023 & 9 & 0,009 & 2,68 & 0,0314 \\
\hline
\end{tabular}

D.E.: Desviación Estándar; E.S. $(\bar{p}) .:$ Error estándar del promedio (hembras + machos); G.L.: Grados de Libertad; $(*)$ Datos transformados según Freeman y Tukey (1950), Kihlberg et al. (1972).

Anexo 2 (continuación)

b) Temperatura $24^{\circ} \mathrm{C}$

\begin{tabular}{|c|c|c|c|c|c|c|c|c|c|c|c|c|c|c|c|c|}
\hline \multirow{2}{*}{ Característica } & \multicolumn{4}{|c|}{ Familia 5} & \multicolumn{4}{|c|}{ Familia 6} & \multicolumn{4}{|c|}{ Familia 7} & \multicolumn{4}{|c|}{ Familia 8} \\
\hline & $\begin{array}{c}n \\
(H+M)\end{array}$ & H-M & $\mathbf{t}$ & $\mathbf{p}$ & $\begin{array}{c}\mathbf{n} \\
(\mathbf{H}+\mathbf{M})\end{array}$ & H-M & $\mathbf{t}$ & $\mathbf{p}$ & $\begin{array}{c}\mathbf{n} \\
(\mathbf{H}+\mathbf{M})\end{array}$ & -M & $\mathbf{t}$ & $\mathbf{p}$ & $\begin{array}{c}\mathbf{n} \\
(\mathbf{H}+\mathbf{M})\end{array}$ & M & $t$ & $\mathbf{p}$ \\
\hline Long & 16 & 0,144 & 13,841 & $<0,0001$ & 13 & $-0,1371$ & $-6,354$ & 0,0001 & 9 & $-0,14$ & $-7,024$ & 0,0002 & 13 & $-0,0323$ & $-1,228$ & 0,2451 \\
\hline $\mathrm{BP}+\mathrm{PHP} *$ & 7 & 0,013 & 3,696 & 0,0141 & 6 & 0,0363 & 2,786 & 0,0495 & 8 & 0,0050 & 1,390 & 0,2138 & 11 & $-0,0154$ & $-5,075$ & 0,0007 \\
\hline PHD* & 15 & $-0,021$ & $-9,942$ & $<0,0001$ & 11 & $-0,0045$ & $-0,694$ & 0,5050 & 9 & 0,0041 & 0,791 & 0,4551 & 11 & $-0,0285$ & -13 & $5<0,0001$ \\
\hline HP* & 15 & $-0,011$ & $-11,081$ & $<0,0001$ & 12 & 0,0170 & 7,538 & $<0,0001$ & 9 & $-0,0183$ & $-9,258$ & $<0,0001$ & 12 & -0 & 67 & 0,0006 \\
\hline $\mathrm{HD}^{*}$ & 16 & $-0,019$ & $-16,039$ & $<0,0001$ & 13 & $-0,0107$ & $-4,266$ & 0,0013 & 9 & 0,0227 & 6,877 & 0,0002 & 12 & $-0,0118$ & $-3,606$ & 0,0048 \\
\hline PSP* & 16 & 0,002 & 1,358 & 0,1959 & 13 & 0,0366 & 10,809 & $<0,0001$ & 9 & 0,0023 & 0,879 & 0,4086 & 12 & 0,0057 & 1,897 & 0,0870 \\
\hline PSD* & 16 & $-0,033$ & $-22,231$ & $<0,0001$ & 12 & $-0,0295$ & $-6,799$ & $<0,0001$ & 9 & 0,0037 & 0,979 & 0,3600 & 12 & $-0,0416$ & $-10,066$ & $6<0,0001$ \\
\hline $\mathrm{SP}^{*}$ & 16 & $-0,014$ & $-14,609$ & $<0,0001$ & 12 & 0,0094 & 4,200 & 0,0018 & 9 & $-0,0003$ & $-0,114$ & 0,9127 & 13 & 0,0033 & 1,411 & 0,1860 \\
\hline PRSD & 16 & $-0,018$ & $-10,766$ & $<0,0001$ & 12 & 0,0373 & 19,484 & $<0,0001$ & 9 & 0,0133 & 5,887 & 0,0006 & 13 & $-0,0061$ & $-2,217$ & 0,0486 \\
\hline ASP* & 16 & $-0,015$ & $-16,139$ & $<0,0001$ & 12 & 0,0467 & 6,143 & 0,0001 & 9 & $-0,0019$ & $-0,500$ & 0,6321 & 13 & 0,0038 & 1,231 & 0,2440 \\
\hline DSD* & 16 & 0,017 & 8,889 & $<0,0001$ & 12 & 0,0743 & 23,057 & $<0,0001$ & 9 & 0,0435 & 14,347 & $<0,0001$ & 13 & 0,0161 & 3,200 & 0,0085 \\
\hline $\mathrm{SCP}^{*}$ & 16 & $-0,032$ & - 28,932 & 001 & 12 & $-0,0089$ & $-3,187$ & 0, & 9 & $-0,0467$ & $-8,331$ & 1 & 13 & $-0,0067$ & $-2,756$ & 0,0187 \\
\hline $\mathrm{PD}^{*}$ & 12 & 0,035 & 16,062 & $<0,0001$ & 12 & 0,0231 & 4,597 & 0,0010 & 9 & 0,0478 & 11,782 & $<0,0001$ & 13 & 0,0527 & 9,230 & $<0,0001$ \\
\hline PP* & 9 & $-0,018$ & $-7,369$ & 0,0002 & 11 & $-0,0158$ & $-3,987$ & 0,0032 & 9 & 0,0037 & 1,608 & 0,1518 & 13 & 0,0053 & 2,310 & 0,0413 \\
\hline $\mathrm{AD}^{*}$ & 9 & $-0,001$ & $-1,231$ & 0,2580 & 7 & 0,0357 & 6,050 & 0,0018 & 7 & 0,0304 & 6,566 & 0,0012 & 12 & 0,0086 & 3,179 & 0,0098 \\
\hline $\mathrm{AP}^{*}$ & 14 & 0,004 & 3,228 & 0,0072 & 11 & 0,0315 & 14,166 & $<0,0001$ & 5 & 0,0037 & 0,265 & 0,8081 & 12 & 0,0017 & 0,698 & 0,5010 \\
\hline $\mathrm{HP} / \mathrm{PHD}^{* *}$ & 10 & $-0,148$ & $-3,948$ & 0,0043 & 8 & 0,0360 & 1,522 & 0,1788 & 8 & $-0,390$ & 11,238 & $3<0,0001$ & 11 & 0,1677 & 7,048 & $<0,0001$ \\
\hline $\mathrm{SCP} / \mathrm{DSD}^{* *}$ & 16 & $-0,078$ & - 22,117 & $<0,0001$ & 12 & $-0,0576$ & 24,818 & $<0,0001$ & 9 & $-0,139$ & 11,487 & 0,00001 & 13 & $-0,019^{\prime}$ & 10,721 & $1<0,0001$ \\
\hline $\begin{array}{l}\text { (SP + PRSD + } \\
\text { ASP)* }\end{array}$ & 16 & $-0,02$ & 11 & 001 & 12 & $-0,04$ & -1 & 11 & 9 & $-0,02$ & 1. & $<0$, & 13 & 0,0246 & 5,052 & 0,0004 \\
\hline $\mathrm{PB} /$ ta-III $2 * *$ & 12 & 0,015 & 10,970 & $<0,0001$ & 11 & 0,0317 & 10,589 & $<0,0001$ & 8 & $-0,0188$ & $-7,893$ & 0,0002 & 10 & 0,0042 & 2,209 & 0,0582 \\
\hline
\end{tabular}


Anexo 2 (Continuación)

c) Temperatura $28^{\circ} \mathrm{C}$

\begin{tabular}{|c|c|c|c|c|c|c|c|c|c|c|c|c|}
\hline \multirow[b]{2}{*}{ Caracter } & \multicolumn{4}{|c|}{ Familia 9} & \multicolumn{4}{|c|}{ Familia 10} & \multicolumn{4}{|c|}{ Familia 11} \\
\hline & $\begin{array}{c}n \\
(H+M)\end{array}$ & $\mathbf{H}-\mathbf{M}$ & $\mathbf{t}$ & $\mathbf{p}$ & $\begin{array}{c}n \\
(H+M)\end{array}$ & H - M & $\mathbf{t}$ & $\mathbf{p}$ & $\begin{array}{c}n \\
(H+M)\end{array}$ & H-M & $\mathbf{t}$ & $\mathbf{p}$ \\
\hline Longitud (mm) & 8 & 0,0400 & 1,084 & 0,3200 & 11 & $-0,0162$ & $-0,741$ & 0,4775 & 9 & 0,4111 & 11,951 & 0,00001 \\
\hline $\mathrm{BP}+\mathrm{PHP}$ & 5 & 0,0235 & 4,368 & 0,0222 & 11 & $-0,0272$ & $-9,835$ & $<0,0001$ & 9 & $-0,0140$ & $-4,278$ & 0,00366 \\
\hline PHD & 8 & $-0,0412$ & $-8,387$ & 0,0002 & 11 & 0,0102 & 3,612 & 0,0056 & 9 & $-0,0406$ & $-9,566$ & 0,00003 \\
\hline HP & 8 & $-0,0127$ & $-4,359$ & 0,0048 & 11 & 0,0035 & 1,493 & 0,1696 & 9 & $-0,0245$ & $-16,142$ & $<0,0001$ \\
\hline HD & 8 & $-0,0219$ & $-5,033$ & 0,0024 & 11 & 0,0208 & 10,646 & $<0,0001$ & 9 & $-0,0319$ & $-9,835$ & $<0,0001$ \\
\hline PSP & 8 & $-0,0044$ & $-1,424$ & 0,2044 & 11 & $-0,0215$ & $-10,048$ & $<0,0001$ & 9 & $-0,0279$ & $-10,883$ & $<0,0001$ \\
\hline PSD & 8 & $-0,0026$ & $-1,195$ & 0,2771 & 11 & 0,0137 & 2,476 & 0,0352 & 9 & $-0,0278$ & $-7,805$ & 0,0001 \\
\hline SP & 8 & $-0,0334$ & $-12,347$ & $<0,0001$ & 11 & $-0,0427$ & $-10,900$ & $<0,0001$ & 9 & $-0,0339$ & $-9,085$ & $<0,0001$ \\
\hline PRSD & 8 & 0,0007 & 0,225 & 0,8298 & 11 & 0,0177 & 7,051 & 0,0001 & 9 & $-0,0188$ & $-6,531$ & 0,0003 \\
\hline ASP & 8 & $-0,0053$ & $-2,110$ & 0,0794 & 11 & $-0,0048$ & $-2,689$ & 0,0248 & 9 & $-0,0189$ & $-5,326$ & 0,0011 \\
\hline DSD & 8 & $-0,0189$ & $-2,867$ & 0,0285 & 11 & 0,0312 & 8,289 & $<0,0001$ & 9 & $-0,0273$ & - 8,516 & $<0,0001$ \\
\hline $\mathrm{SCP}$ & 8 & 0,0031 & 1,308 & 0,2386 & 11 & $-0,0140$ & $-7,040$ & 0,0001 & 9 & $-0,0300$ & $-15,374$ & $<0,0001$ \\
\hline PD & 8 & $-0,0117$ & $-3,580$ & 0,0116 & 11 & 0,0952 & 18,677 & $<0,0001$ & 9 & $-0,0173$ & - 7,430 & 0,0002 \\
\hline PP & 7 & $-0,0051$ & $-1,998$ & 0,1022 & 9 & $-0,0895$ & $-27,280$ & $<0,0001$ & 8 & $-0,0075$ & $-5,554$ & 0,0014 \\
\hline $\mathrm{AD}$ & 5 & - & - & - & 8 & 0,0220 & 6,068 & 0,0009 & 6 & $-0,0323$ & $-5,952$ & 0,0040 \\
\hline $\mathrm{AP}$ & 5 & - & - & - & 8 & $-0,0221$ & $-6,056$ & 0,0009 & 6 & $-0,0426$ & $-10,791$ & 0,0004 \\
\hline HP/PHD & 7 & 0,1716 & 4,019 & 0,0101 & 11 & $-0,0297$ & $-3,305$ & 0,0092 & 9 & 0,1276 & 4,390 & 0,0032 \\
\hline SCP/DSD & 8 & 0,0169 & 2,770 & 0,0324 & 11 & $-0,0374$ & $-23,557$ & $<0,0001$ & 9 & 0,0005 & 0,139 & 0,8935 \\
\hline $\mathrm{SP}+\mathrm{PRSD}+\mathrm{ASP}$ & 8 & $-0,0275$ & $-9,337$ & 0,0001 & 11 & $-0,0448$ & $-13,416$ & $<0,0001$ & 9 & 0,0438 & 17,197 & $<0,0001$ \\
\hline $\mathrm{PB} /$ ta-III2 & 3 & - & - & - & 8 & 0,0193 & 7,379 & 0,0003 & 8 & $-0,0749$ & $-12,136$ & $<0,0001$ \\
\hline
\end{tabular}

